\title{
Stress analysis of three-dimensional contact problems using the boundary element method
}

DOI:

10.1016/S0955-7997(98)00050-2

\section{Document Version}

Accepted author manuscript

Link to publication record in Manchester Research Explorer

\section{Citation for published version (APA):}

Segond, D., \& Tafreshi, A. (1998). Stress analysis of three-dimensional contact problems using the boundary element method. Engineering Analysis with Boundary Elements, 22(3), 199-214. https://doi.org/10.1016/S09557997(98)00050-2

\section{Published in:}

Engineering Analysis with Boundary Elements

\section{Citing this paper}

Please note that where the full-text provided on Manchester Research Explorer is the Author Accepted Manuscript or Proof version this may differ from the final Published version. If citing, it is advised that you check and use the publisher's definitive version.

\section{General rights}

Copyright and moral rights for the publications made accessible in the Research Explorer are retained by the authors and/or other copyright owners and it is a condition of accessing publications that users recognise and abide by the legal requirements associated with these rights.

\section{Takedown policy}

If you believe that this document breaches copyright please refer to the University of Manchester's Takedown Procedures [http://man.ac.uk/04Y6Bo] or contact uml.scholarlycommunications@manchester.ac.uk providing relevant details, so we can investigate your claim.

\section{OPEN ACCESS}




\title{
STRESS ANALYSIS OF THREE-DIMENSIONAL CONTACT PROBLEMS USING THE BOUNDARY ELEMENT METHOD
}

\author{
D. SEGOND The School of Engineering, University of Manchester \\ A. TAFRESHI Aerospace Engineering Division, \\ The School of Engineering, University of Manchester
}

This paper presents a technique based on the boundary element method (1), to analyse threedimensional contact problems. The formulation is implemented for the frictionless and infinite friction conditions. Following a review of the basic nature of contact problems, the analytical basis of the direct formulation of the boundary element method is described. The numerical implementation employs linear triangular elements for the representation of the boundary and variables of the bodies in contact. Opposite nodal points in similar element pairs are defined on the two surfaces in the area which are expected to come into contact under the increasing load. The use of appropriate contact conditions enables the integral equations for the two bodies to be coupled together. Following an iteration procedure, the size of the contact zone is determined by finding a boundary solution compatible with the contact conditions. Different examples have been analysed in order to verify the applicability of the proposed method to various contact situations. The results have been compared with those obtained using the finite element method in conjunction with the ABAQUS (2) and IDEAS (3) packages which are shown to be in good agreement.

\section{INTRODUCTION}

Because of the non-linear nature of the contact problem(geometry and friction) analytical solutions only exist for simple geometries with frictionless contact(4-6). Complex geometrical shapes in real engineering problems, the wide variety of boundary conditions and also the nonlinear nature of contact have made it necessary to use numerical methods. The finite element method(FEM) (7-9) and boundary element method(BEM)(10-21) are the techniques currently 
employed. The first analysis of three-dimensional contact problems using constant elements was presented by Paris et al (11). This study was developed for the three possible contact conditions: conforming, advancing or receding, confined to frictionless surfaces. It was later extended to the case with friction using an incremental procedure of application of the load and an iterative procedure to determine the sliding direction (12).

For linear elastic problems the BEM offers significant advantages over the FEM. Using the BEM the dimensionality of the problem is effectively reduced by one, so only the boundary has to be discretized. This is very efficient as in the majority of stress analysis problems the maximum stress occurs on the boundary and therefore it is not necessary to compute internal stresses and displacements (22-23). This reduces the computational cost and capacity producing easier model preparation. The BEM offers continuous interior modelling within the solution domain giving high resolution of stresses and displacements at the boundary. Using the FEM it is not possible to apply the contact conditions directly. This means that contact pressure does not appear as an independent variable in the formulation and penalty methods or mathematical programming techniques have to be used to overcome this problem. Using the BEM the variables involved in the contact zone such as stresses and displacements are handled directly in the formulation. However, the BEM has some drawbacks. The BEM is not suitable when information is needed for a large number of internal points. It is not suitable for non-linear problems, such as elastoplastic and large strain analysis problems because the non-linearities no longer allow the partial differential equations to be fully transformable to the surface of the body and some form of domain discretization is therefore required. However, it is suitable for the contact problems because the non-linearities in contact occur at the boundary.

This work is directed specifically toward the solution of three-dimensional frictionless contact problems using the boundary element method. Indeed some of the unknowns involved in the contact are in many problems not greatly affected by the presence of friction such as the size of the contact zone, the evolution of the contact pressures, etc.. Furthermore the solution of a frictionless case can be the first step in a friction analysis, to determine qualitatively some of the information of the frictional problem. Here linear triangular elements are employed for the analysis which give a better accuracy than constant triangular elements used elsewhere (11-12). 
Segond, D. \& Tafreshi, A. Oct 1998 In : Engineering Analysis with Boundary Elements. 22, 3, p. 199-214 16 p.

\section{NOTATION}

$[A]^{(k)} \quad$ Matrix of coefficients for body $k$

$C_{y j}(P) \quad$ The limiting value of the surface integral of $T_{i j}(p, Q)$

$d(b, c) \quad c$ th node of the $b$ th surface element

E Young's modulus

$J(\xi) \quad$ Jacobian of transformation from global Cartesian coordinates

to intrinsic coordinates of element

$L^{m}(\eta) \quad$ Linear shape functions in intrinsic coordinates.

$M \quad$ Total number of surface elements

$n_{i} \quad$ Unit normal at a boundary point

$N^{c}(\xi) \quad$ Quadratic shape function corresponding to the $c$ th node of the element

$p(x) \quad$ Load point within the elastic domain $R$

$P(x) \quad$ Load point at the surface of the elastic domain

$q(x) \quad$ Field point within the elastic domain $R$

$Q(x) \quad$ Field point at the surface of the elastic domain

$r \quad$ Distance between field and load points

$R \quad$ Elastic domain

$t_{i} \quad$ Traction

$T_{i j}(p, q) \quad$ The $i$ th component of the traction vector at point $q(x)$ due to a unit point load in the $j$ th direction at $p(x)$

$[T]^{(k)} \quad$ Matrix of coefficients for nodal displacements of body $k$

$u_{i} \quad$ Displacement

$U_{y}(p, q) \quad$ The $i$ th component of the displacement vector at point $q(x)$ due

to a unit point load in the jth direction at $p(x)$ 


$\begin{array}{ll}{[U]^{(k)}} & \text { Matrix of coefficients for nodal tractions of body } k \\ x_{i} & \text { Rectangular Cartesian coordinates } \\ x_{i}^{c} & \text { Cartesian coordinates of the } c \text { th node of element } \\ \alpha, \beta & \text { Cosine direction of the unit normal } \\ \delta_{i j} & \text { Kronecker delta } \\ \varepsilon_{i j} & \text { Strain tensor } \\ \eta & \text { Intrinsic coordinates of triangular element } \\ \sigma_{i j} & \text { Stress tensor } \\ \zeta_{i} & \text { Coordinates of load point } \\ \mu & \text { Coulomb's coefficient of friction } \\ v & \text { Poisson's ratio } \\ \Delta_{l} & \text { Distance between a node-pair that can be in contact }\end{array}$

\section{GENERAL DESCRIPTION OF CONTACT PROBLEMS}

Contact problems differ from other elastic problems since the contact area is not explicitly specified, and its extent must be determined as part of the solution of the problem. If friction is considered to exist in the contact zone, the contact problem is thermodynamically irreversible. Consequently the final state of displacements and tractions depends on the entire loading history and the problem must be solved by applying the load incrementally, with the assumption that the behaviour during each load increment or step is approximately irreversible. However, for a frictionless contact the solution can be iterative where no load increment is required.

In general, different kinds of contact can result, depending on the geometry of the contacting bodies. Two different types of contact are met : infinite friction(sticking) and frictionless(conforming, receding and advancing). For the conforming contact, the contact region does not change upon loading. For the receding contact, the contact region reduces with the application of the load. Finally, for the advancing contact, the contact region increases depending on the geometry, the material properties of the bodies involved in the contact and the amplitude of the external forces. 
In a general contact problem several regions can be recognised. Consider two linear elastic bodies, A and B, in a Cartesian co-ordinates system which are in contact(see Fig. 1). The boundary of each body is divided into two complementary zones: contact zone $\left(S_{c}^{k}\right)(k=A, B)$ and non-contact zone $\left(\mathrm{S}_{1}\right)(\mathrm{k}=\mathrm{A}, \mathrm{B})$. The contact zone can be divided into: separation region and contact region. In the separation region the boundaries have not come into contact or, if previously in contact, have separated as a result of the load application. In the contact region the boundaries have come into contact or remain in contact. The non contact zone can be subdivided into regions with prescribed displacements $\left(\mathrm{S}_{\mathrm{lu}}^{\mathrm{k}}\right)$ and prescribed tractions $\left(\mathrm{S}_{\mathrm{lt}}^{\mathrm{k}}\right)$.

In order to determine the correct contact zone, a potential contact zone must be initially assumed $\left(S_{c p}\right)$. The elements belonging to this zone must always be estimated in excess. In contrary the elements selected to form the initial contact area $\left(S_{c o}\right)$ must be limited to a minimum (the minimum number of elements in this initial contact area is one). When all the conditions of continuity and stability are met in the contact zone, the iterative procedure is terminated, and the final contact zone $\left(S_{c}\right)$ is reached. For each class of contact, described above, an iterative strategy is required. This one depends upon the selection of the potential and initial contact zones.

In the case of a conforming contact $S_{c}=S_{c o}=S_{c p}$, where $S_{c}$ and $S_{l}^{k}(k=A, B)$ are known, therefore, the solution is obtained by solving directly the system of equation, once the boundaries have been discretised. In this case only one iteration is needed.

For an advancing contact problem $S_{c o}$ is less than $S_{c}$. $S_{c}$ is not previously known because it depends on the geometry, the mechanical properties of the bodies involved in the contact and on the amplitude of the external loads. $S_{c p}$ is defined in excess and is used for the first iteration. Since the potential contact zone is greater than the final contact area, each iteration allows to check in all contacting element pairs if tensile stresses have been generated. The detection of tensile stresses in contacting element is an indication that the contact area is too large for the given load, and these elements must be released from contact in the next iteration. 
Segond, D. \& Tafreshi, A. Oct 1998 In : Engineering Analysis with Boundary Elements. 22, 3, p. 199-214 16 p.

For receding contact problems the question is relatively similar to the advancing case. $S_{c p}$ is larger than $S_{c}$ and the iteration consists of checking the edge overlap. The displacements of the pairs of elements just outside $S_{c o}$ are checked to determine whether overlap has occurred. The presence of overlap signifies that the contact area is too small for the given load, and the overlapped element pairs must be included in the contact area in the next iteration. However it has been shown by Keer et al. (24) that $S_{c}$ depends only on the relative values of the elastic constants $\left(G^{k}\right.$ and $\left.\nu^{k}, k=A, B\right)$ of the bodies in contact.

The infinite friction contact problem is solved in a similar way to the conforming contact.

Figure 2 shows an algorithm for automatic iterations. In every configuration the definition of the initial and potential contact zones must be as accurate as possible in order to minimise the number of iterations. A quick convergency test is usually obtained by taking $S_{c}=S_{c p}$. The iteration procedure is completed when the conditions of edge overlap and tensile stress, described in formulas (1a) and (1b) are satisfied.

$$
\begin{aligned}
& t_{s}^{k} \leq 0 \quad(k=A, B) \\
& U_{1}^{A}+U_{1}^{B} \leq \Delta_{1}
\end{aligned}
$$

If condition (la) is satisfied all the nodes assumed to belong to the contact zone are in compression (the normal tension at the nodes near, but outside the contact zone is known and is usually zero). If condition ( $\mathrm{lb}$ ) is satisfied no interpenetrations occur at the nodes outside, but near, the contact zone $S_{c}$. Inside $S_{c}$ normal displacements are related to equation (1b). The term $\Delta_{l}$ corresponds to the distance between two nodes (node-pair) that can be in contact after the application of the load.

\section{FORMULATION OF THE BOUNDARY INTEGRAL EQUATION}

Starting from the Betti's reciprocal theorem and using the fundamental solution of Navier's equations, the somigliana identity is obtained. This integral equation for displacement is 
$u_{i}(p)=\int_{S} t_{j}(Q) U_{i j}(p, Q) d s(Q)-\int_{s} u_{j}(Q) T_{i j}(p, Q) d s$

$p$ is a point within region of analysis, $u_{j}$ and $t_{i}$ are the displacement and traction vectors, respectively. The kernel functions $U_{i j}(p, Q)$ and $T_{i j}(p, Q)$ are, respectively, the displacements and traction components in the $x_{i}$ coordinate direction at point $Q$ due to a unit point load in the $x_{j}$ direction at point $\mathrm{p}$. The second-order tensors, $\mathrm{U}_{\mathrm{ij}}$ and $\mathrm{T}_{\mathrm{ij}}$, are given by

$$
\begin{array}{r}
U_{i j}(p, q)=\frac{1}{16 \pi \mu(1-v)}\left(\frac{1}{r}\right)\left[(3-4 v) \zeta_{i j}+r_{, i} r_{, j}\right] \\
T_{i j}(p, q)=-\frac{1}{8 \pi(1-v)}\left(\frac{1}{r^{2}}\right)\left\{\frac{\partial}{\partial n}\left[(1-2 v) \delta_{i j}+3 r_{, i} r_{, j}\right]\right. \\
\left.-(1-2 v)\left(n_{j} r_{i j}-n_{i} r_{, j}\right)\right\}
\end{array}
$$

and the normal $n_{i}$ is also evaluated at $Q$. Taking $p$ to a boundary point $P$, the following boundary integral equation $(\mathrm{BIE})$ is obtained

$C_{i j} u_{j}(P)+\int_{S} T_{i j}(P, Q) u_{j}(Q) d s=\int_{S} U_{i j}(P, Q) t_{j}(Q) d s$

where constant $C_{i j}$ depends on the local geometry of the boundary at point $P$, whether it is smooth or sharp.

For two bodies in contact Equation (5) is employed individually for each body involved. In general, the equations can be written as:

$C_{i j}{ }^{(k)} u_{j}(P)+\int_{S} T_{i j}^{k}(P, Q) u_{j}^{k}(Q) d s=\int_{S} U_{i j}{ }^{k}(P, Q) t,{ }^{k}(Q) d s$

where $k(A, B)$ represents the bodies in contact. These equations are coupled together via the relations between the displacements and tractions in the contact areas. 


\section{NUMERICAL FORMULATION OF THE BOUNDARY INTEGRAL EQUATIONS}

For the numerical solution to BIE, linear or parabolic triangular and/or quadrilateral elements can be employed for discretization of the boundary (25-26). For parabolic elements in the contact region one extra node at the centroid of each element is necessary. This is because for parabolic elements compressive surface pressure can generate tensile nodal forces at the comer nodes and the contact elements will be ignored whenever tensile forces are applied. Linear elements do not exhibit tensile equivalent nodal forces for compressive surface pressures and can be used freely.

In the computer program developed in the present work, the surface is represented by three-node linear triangular elements, and the representation of geometry and unknowns are in terms of linear shape functions of intrinsic coordinates of the elements. Let there be a total of $\mathrm{M}^{(\mathrm{k})}$ linear triangular elements with a total of $\mathrm{g}^{(k)}$ nodes over the boundary of the body $\mathrm{k}$. $\mathrm{d}\left(\mathrm{b}^{(\mathrm{k})}, \mathrm{c}\right)$ represent the cth node of the bth element $\left(c=1,3\right.$ and $\left.b^{(k)}=1, M^{(k)}\right)$. Substitution of the parametric representation of displacements $u_{i}^{(k)}$ and tractions $t_{i}^{(k)}$ into Eq.(6) for each of the $g^{(k)}$ nodes results in the following set of simultaneous equations:

$$
\begin{aligned}
c_{i j}\left(P_{n}\right) u_{j}^{(k)}\left(P_{n}\right)+\sum_{b^{(k)}=1}^{M^{(k)}} \sum_{c=1}^{3} u_{j}\left(P_{d\left(b^{(k)}, c\right)}\right) \int_{S_{b}^{(k)}} T_{i j}^{(k)}\left(P_{n}, Q(\xi)\right) N^{c}(\xi)|J(\xi)| d \xi \\
\quad=\sum_{b^{(k)}=1}^{M^{(k)}} \sum_{c=1}^{3} t_{j}\left(P_{d\left(b^{(k)}, c\right)}\right) \int_{S_{b}^{(k)}} U_{j}^{(k)}\left(P_{n}, Q(\xi)\right) N^{c}(\xi)|J(\xi)| d \xi
\end{aligned}
$$

where $[\mathrm{T}]^{(\mathrm{k})}$ and $[\mathrm{U}]^{(\mathrm{k})}$ are the matrices containing the coefficients of the equations of nodal collocation of body $k$.

Eq. 7 represents a set of $3 \mathrm{~g}^{(\mathrm{k})}$ equations with $6 \mathrm{~g}^{(\mathrm{k})}$ nodal values of displacements and tractions of body $k$. Except in the contact area, either the displacements or tractions are prescribed in a piecewise fashion along the boundary. As a result, half of the boundary nodal values are known. If there is a total of $\mathrm{g}_{c}{ }^{(k)}$ contact nodes, these would yield $3 \mathrm{~g}_{c}{ }^{\left({ }^{(k)}\right.}$ equations with $6 \mathrm{~g}_{\mathrm{c}}{ }^{(\mathrm{k})}$ 
unknown values of displacements and tractions. Of the total number of unknowns in Eq.(7), $3 \mathrm{~g}^{(\mathrm{k})}-3 \mathrm{~g}_{\mathrm{c}}{ }^{(k)}$ nodal values are known, and a set of $3 \mathrm{~g}^{(\mathrm{k})}$ equations with $3 \mathrm{~g}^{(\mathrm{k})}+3 \mathrm{~g}_{\mathrm{c}}{ }^{(\mathrm{k})}$ unknowns result. However, coupling the equations in the contact area, by substituting the equivalent values based on contact conditions, the $3 \mathrm{~g}_{\mathrm{c}}{ }^{(k)}$ extra unknowns would be solved for.

To be able to use standard solvers, the $[\mathrm{T}]$ and $[\mathrm{U}]$ matrices must be rearranged such that all the unknown variables are on the right hand side and all the known variables are on the left hand side which will result in the following system of algebraic equations:

$$
[A] \underline{\underline{z}}=\underline{b}
$$

Here, $\mathrm{A}$ is the final coefficient matrix; $\mathrm{z}$ is a vector containing the unknown variables (whether displacements or tractions). The second member vector $b$ consists of all known values multiplied by corresponding matrix elements.

However, for the nodes in contact area, neither the displacements nor the tractions are known. Depending on the contact conditions, there are certain relationships between the displacements and tractions of node pairs. For example, in the case of a complete sticking contact, the matrix equations of (8) can be arranged as follows:

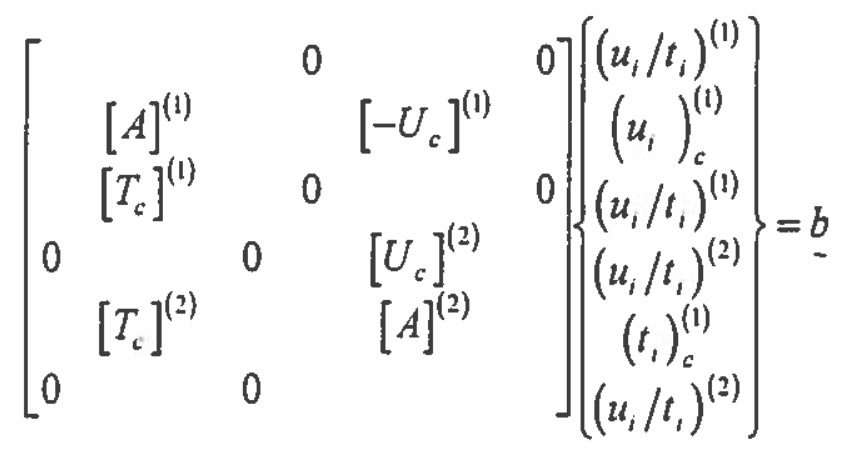

where $[A]^{(k)}$ is the final matrix of unknown coefficients for body $k$ which also contains the coefficients of displacements in the contact area (the matrix $\left.\left[\mathrm{T}_{c}\right]^{(\mathrm{k})}\right) .\left[\mathrm{U}_{\mathrm{c}}\right]^{(\mathrm{k})}$ is a matrix which is part of $[\mathrm{U}]^{(k)}$, the coefficient matrix multiplying tractions for the nodes in the contact area. For other possible cases refer to Reference (1). 


\subsection{LINEAR TRIANGULAR ELEMENT}

In a typical linear triangular element the two local intrinsic coordinates, $\zeta_{1}$ and $\zeta_{2}$, are attached to two edges of the triangle and vary from 0 to 1 . Therefore, the geometry of each element is defined by the coordinates of its three nodes using linear shape functions as follows:

$$
\begin{aligned}
& N_{1}=1-\xi_{1}-\xi_{2} \\
& N_{2}=\xi_{1} \\
& N_{3}=\xi_{2}
\end{aligned}
$$

In Eq. (7) where $P_{n}$ is a node of $S_{b}{ }^{(k)}$, the integral varies very rapidly and special techniques must be used to remove the strong singularities that are present. The $U_{i j}$ and $T_{i j}$ kernels are singular because they contain terms of the order $1 / \mathrm{r}$ and $1 / \mathrm{r}^{2}$, respectively. The removal of these singularities can be accomplished through the use of the shape functions by defining a new set of local intrinsic coordinates, $\eta_{1}$ and $\eta_{2}$ with their origin at the centre of the element(see Fig. 3 ). The new triangular element is considered as degenerate quadrilateral, where the coordinates $\eta_{a}$ are defined in terms of $\zeta_{\mathrm{a}}$ by the relations:

$$
\xi_{a}(\eta)=L^{m}(\eta) \xi_{a}^{(m)} \quad m=1,4
$$

where $L_{m}(\eta)$ are the linear shape functions similar to the shape functions of a linear quadrilateral element(27).

\subsection{TREATMENT OF BOUNDARY CONDITIONS}

Four categories of boundary conditions are considered in the programme used for the analysis in this paper, namely: I) prescribed tractions II) uniform stresses or pressure III) prescribed displacements IV) contact conditions. However, two other specific cases are also considered; constrained elements in specific direction and point constraints. See reference (1).

When the tractions are prescribed over a boundary element, the nodal values specified at its three nodes are simply multiplied to the corresponding elements of $[\mathrm{U}]^{(k)}$. Tractions are prescribed at every node of each element, so two elements with non-unique tractions at a common node, could come together. Hence non-unique tractions could be prescribed. When 
uniform stresses are prescribed over a boundary element, the nodal tractions are calculated from the normals at the nodes and the given stresses. They are then treated as traction boundary conditions. The adjacent elements may have different prescribed stresses, therefore, non-unique tractions at the connecting node may arise which must be treated accordingly. When the displacements are prescribed over a boundary element, the nodal values specified at its three nodes are simply multiplied by the corresponding elements of $\left[T^{(k)}\right.$ and then subtracted from the second member $b$. The corresponding elements of matrix $[U]^{(k)}$ with their signs reversed are replaced with the coefficients (matrix $[\mathrm{A}]^{(k)}$ ) of the unknown tractions at the same nodes. When a constrained element is next to an element with prescribed tractions, it must always be ensured that the contribution to the traction coefficients at the node joining the two elements are treated separately. If a constrained element is at a comer, then the three unknowns for the comer node should be defined properly. No special consideration is required if the other element has prescribed tractions.

If in the boundary conditions, some displacements in one or both directions are not specified, for both bodies involved in the contact, a singular matrix [A] may arise. In such cases the determinant of $[A]$ is zero, and physically, this means that an infinite number of displacements could arise from different degrees of rigid body translation or rotation. In such cases a datum is always required, through imposing a constrained node in that direction. Only one node is enough for the bodies in contact. For each such constrained node, the whole row and column of the matrix corresponding to that node is made equal to zero, with the exception of the diagonal coefficient which is set equal to unity. The corresponding second member of the matrix is then replaced by the value of the displacement constraint.

\subsection{TREATMENT OF CONTACT CONDITIONS}

In the case of two bodies coming into contact, corresponding elements on the opposite surfaces of the contact interface constitute element pairs and the nodes of these element pairs constitute the node-pairs. Inside the contact zone the discretisation is made in such a way that both bodies contain the same number of elements and nodes. Furthermore the elements from 
each surface of the interface must have the same geometries and dimensions in the undeformed state.

Referring to Figure 4 , the components of the unit normal vector in the global coordinate system are $n_{1}$ with $(i=1,3)$. In order to couple the governing equations for the bodies in contact, a local system of reference for the node-pairs is defined. Having the components of the unit normal vector in the global coordinate system, the two other directions are defined to be orthonormal and to belong to the plane tangent to the boundary. The components of the unit normal vector are the cosine directions of this vector.

In the case where the normal unit vector was defined in the plane $x y$, the tangential directions can be easily defined:

$$
\left[\begin{array}{l}
n \\
t_{1} \\
t_{2}
\end{array}\right]=\left[\begin{array}{ccc}
\alpha & \beta & 0 \\
-\beta & \alpha & 0 \\
0 & 0 & 1
\end{array}\right]\left[\begin{array}{l}
x_{1} \\
x_{2} \\
x_{3}
\end{array}\right]
$$

in such a way that the following relationships are satisfied: first the norm of the vectors is equal to 1 (Eq. 13a) and second the system formed by the three vector is orthonormal(14b).

$$
\begin{aligned}
& |n|=\sqrt{\alpha^{2}+\beta^{2}}=1 \\
& n=t_{1} \wedge t_{2}
\end{aligned}
$$

At the nodes which connect adjacent elements, different values $n_{i}$ may be achieved. However, the values are very near to each other, especially in the contact area, where a very fine mesh is expected.

Two nodes one from each side of the interface which make a node-pair when in contact, have the same unit normal, but with opposite sign. Similarly one of the tangential direction must be identical for the node pair. For node-pairs which are expected to come into contact at different stages of the analysis, a mean absolute value of normal is calculated which is needed when the relative displacement between the nodes is measured. In reference to Figure 5 , the mean absolute normal is defined as direction 1 which coincides with the distance $\Delta_{1}$, 
between the two nodes that can be in contact after the application of the load. The positive sense in each body is taken again as outward to the respective boundary. According to this, it is assumed that contact appears when the two nodes that are going to contact reach a common plane, not necessarily at the same point, normal to direction $I$. Here two contact conditions are considered; stick contact node pairs and frictionless contact.

In the case of stick contact node pairs it is assumed that all node-pairs are stuck together, so there is no relative slip between node-pairs. It means for a sticky node-pair a and b of bodies 1 and 2 :

$$
\begin{aligned}
& u_{i}^{a}+x_{i}^{a}=u_{i}^{b}+x_{i}^{b} \quad \text { with }(\mathrm{i}=1,3) \\
& t_{j}^{a}+t_{i}^{b}=0 .
\end{aligned}
$$

For the frictionless contact the tangential surface traction is always zero. For frictionless node-pairs, all the relations which hold between the nodal tractions and displacements for the Coulomb slip contact condition are applied, except that the value of $\mu$, the "Coulomb coefficient of friction" is set to zero.

Although it is necessary to consider a local coordinate system for the node-pairs in the contact area, in order to express the contact conditions, these relations must be set globally to form the matrix equations. Assume a node-pair $a$ and $b$ situated on bodies $I$ and 2 , respectively, with their unit normal in the plane $x y$. Therefore, if $t_{x}, t_{y}$ and $t$ are global tractions of nodes a and $b$, the local tractions are written as:

$$
\left[\begin{array}{c}
t_{t 1}^{a} \\
t_{t 2}^{a} \\
t_{n}^{a}
\end{array}\right]=\left[\begin{array}{ccc}
-\beta & \alpha & 0 \\
0 & 0 & 1 \\
\alpha & \beta & 0
\end{array}\right]\left[\begin{array}{l}
t_{x} \\
t_{y} \\
t_{z}
\end{array}\right],\left[\begin{array}{l}
t_{t 1}^{b} \\
t_{t 2}^{b} \\
t_{n}^{b}
\end{array}\right]=\left[\begin{array}{ccc}
-\beta & \alpha & 0 \\
0 & 0 & -1 \\
-\alpha & -\beta & 0
\end{array}\right]\left[\begin{array}{l}
t_{x} \\
t_{y} \\
t_{z}
\end{array}\right]
$$

where $\alpha$ and $\beta$ are the cosine directions of the unit normal.

In the Coulomb slip condition the values of the tangential tractions $t_{11}$ and $t_{12}$ are known as a function of $t_{n}$. In the local coordinate:

$$
t_{t i}= \pm \mu t_{n} \quad \text { with }(i=1,2)
$$


Since in the matrix equation the relations must be set globally, with $\mu=0$ for frictionless contact, the following relations are obtained:

$$
\begin{aligned}
& t_{x}=\frac{\alpha}{\beta} t_{y} \\
& t_{z}=0
\end{aligned}
$$

Care must be taken when the unit normal is defined only in the $x$-direction and must be treated accordingly in order to avoid a division by zero.

It is assumed that slip is in the local tangential directions. For values of $\Delta u_{s 1}$ and $\Delta u_{s 2}$, the amounts of slip in the tangential directions between node-pair $a$ and $b$, the following displacement relations can be written in local coordinates.

$$
\begin{aligned}
& u_{i i}^{a}=u_{i i}^{b}+\Delta u_{s i} \quad \text { with }(i=1,2) \\
& u_{n}^{a}=u_{n}^{b}
\end{aligned}
$$

If these relations are translated into global coordinates, then:

$$
\begin{aligned}
& u_{x}^{a}+x^{a}=u_{x}^{b}+x^{b}-\beta \cdot \Delta u_{s 1} \\
& u_{y}^{a}+y^{a}=u_{y}^{b}+y^{b}+\alpha \cdot \Delta u_{s 1} \\
& u_{z}^{a}+z^{a}=u_{z}^{b}+z^{b}+\Delta u_{s 2}
\end{aligned}
$$

In the matrix equation $u_{x}^{a}$ and $u_{x}^{b}$ are solved independently, and their solution would yield the amount of slip $\Delta u_{s 1}$ :

$$
\Delta u_{\mathrm{s} 1}=\frac{u_{x}^{a}+x^{a}-u_{x}^{b}-x^{b}}{-\beta}
$$

$u_{y}^{b}$ is solved in terms of $u_{x}^{a}, u_{x}^{b}, u_{y}^{a}$ using equation (19), to give:

$$
u_{y}^{b}=u_{y}^{a}+y^{a}-y^{b}-\frac{\alpha}{\beta}\left(u_{x}^{a}+x^{a}-u_{x}^{b}-x^{b}\right)
$$

In the matrix equation $u_{z}^{a}$ and $u_{:}^{b}$ are solved independently. The coefficients of $u_{x}^{a} \cdot u_{y}^{a}, u_{z}^{a}, u_{x}^{b}, u_{z}^{b}$ remain unreplaced. The corresponding coefficients of $u_{y}^{b}$ are added or subtracted from the corresponding columns related to $u_{x}^{a}, u_{y}^{a}, u_{x}^{b}$ according to relation (21) at 
the same rows. The multiplied known values are transferred to the right hand side and are subtracted from the corresponding elements of $b$. The coefficients corresponding to $t_{y}^{a}$ and $t_{x}^{o}$ are added together, multiplied by the scale factor and with the sign changed, transferred to the column previously occupied by $u_{y}^{b}$. This is similarly performed for the coefficients of $t_{y}^{b}$ and $t_{x}^{b}$, but without changing the sign, as they now correspond to $-t_{y}^{a}$ and $-t_{x}^{a}$, respectively. Hence the final form of matrix $[A]$ is obtained in the form given by equation 22 .

\section{Corresponding coefficients} to $\left(u_{i}\right)_{c}^{(1)}$ from coefficients for $\left(u_{y}\right)_{c}^{(2)}$ and $\left(u_{z}\right)_{c}^{(2)}$

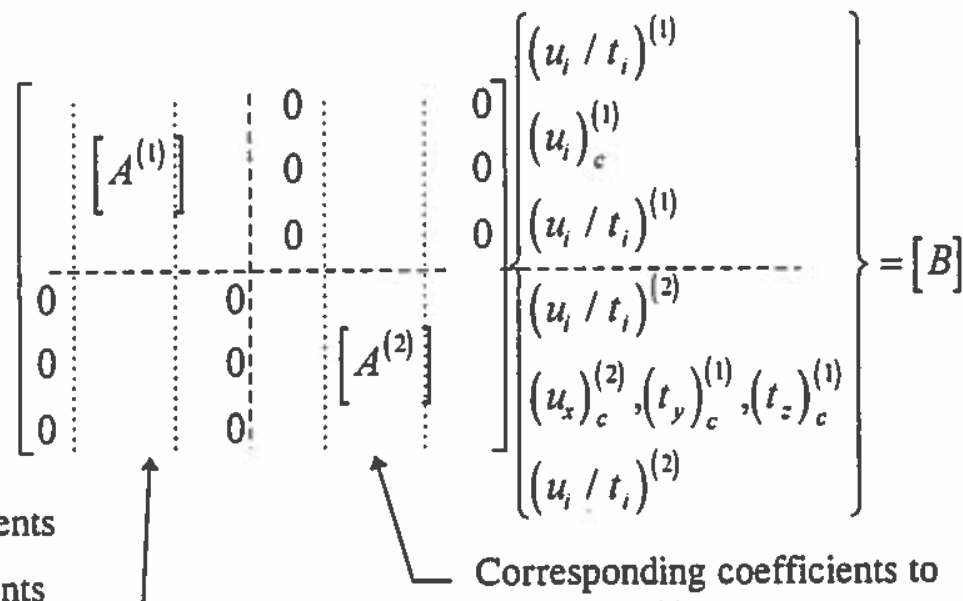

$$
\begin{aligned}
& \left(u_{x}\right)_{c}^{(2)},\left(t_{y}\right)_{c}^{(1)},\left(t_{z}\right)_{c}^{(1)} \text { from coefficients for } \\
& \left(u_{x}\right)_{c}^{(2)},\left(t_{i}\right)_{\varepsilon}^{(1)} \text { and }\left(t_{i}\right)_{c}^{(2)}
\end{aligned}
$$

The unknown vector $z$ now contains $u_{x}^{a}, u_{y}^{a}, u_{z}^{a}, u_{x}^{b}, t_{y}^{a}, u_{z}^{b}$ for the node-pair $a$ and $b$. Having determined the solution, they can be sorted into the tractions and displacements in the contact area for every node of the two bodies.

Where a region of contact comes to an end, at the last node there is always a chance of nonunique tractions. This happens when the next element is subject to a prescribed traction boundary condition. Also, care must be taken when the next element is a constrained element, as happens in symmetric contact problems. In such cases continuity of the displacements must be preserved by applying the known values of the displacements. During the computational procedure relative to the treatment of the boundary conditions when a node is checked to belong to a plane of symmetry as well as to the contact area, it is firstly treated as a prescribed 
displacement and since it belongs to the contact area the coefficients are rearranged to satisfy the contact relationships.

\section{EVALUATION OF THE SURFACE STRESS TENSOR}

In the present work, the stresses are calculated directly from the computed displacements and tractions by simple differentiation using the shape functions. A local orthogonal system of axis is introduced. Let $\mathrm{m}_{\mathrm{lj}}$ and $\mathrm{m}_{2 \mathrm{j}}$ be the unit vectors in the coordinate directions $\zeta_{1}$ and $\zeta_{2}$, respectively, and $m_{3 j}$ the unit normal to the element. If $u_{i j}$ are the direction cosines of this orthogonal system of axes in the global Cartesian system, then the local orthogonal system is defined by taking $\mu_{1 j}=m_{1 j}, \mu_{3 j}=m_{3 j}$ and $\mu_{2 j}$ the vector product of $m_{1 j}$ and $m_{3 j}$. Further, let, $\overline{\mathrm{u}}_{\mathrm{i}}, \overline{\mathrm{e}}_{\mathrm{ij}}, \bar{\sigma}_{\mathrm{ij}}$ and $\overline{\mathrm{t}}_{\mathrm{i}}$ be the displacements, strains, stresses and tractions, respectively, in this orthogonal system defined by $\mu_{\mathrm{ij}}$. The displacements in the orthogonal tangential directions are thus given by:

$$
\bar{u}_{\alpha}(\xi)=N^{c}(\xi) u_{j}^{c} \mu_{a j}
$$

Differentiating Eq.(23) with respect to the local orthogonal coordinates in the surface tangential directions, $\mu_{1 j}$, $\mu_{2 j}$, the tangential strains in this local system of coordinates are obtained and using Hooke's law, the stress tensor $\sigma_{\mathrm{ij}}$ may be obtained from the tractions and these surface strains:

$$
\begin{aligned}
& \bar{\sigma}_{11}=\frac{E}{1-v^{2}}\left(\bar{e}_{11}+v \bar{e}_{22}\right)+\frac{v}{1-v} \bar{t}_{3} \\
& \bar{\sigma}_{12}=\bar{\sigma}_{21}=\frac{E}{2(1+v)} \bar{e}_{12} \\
& \bar{\sigma}_{22}=\frac{E}{1-v^{2}}\left(\bar{e}_{22}+v \bar{e}_{11}\right)+\frac{v}{1-v} \bar{t}_{3} \\
& \bar{\sigma}_{33}=\bar{t}_{3} \\
& \bar{\sigma}_{32}=\bar{\sigma}_{23}=\bar{t}_{1} \\
& \bar{\sigma}_{31}=\bar{\sigma}_{13}=\bar{t}_{1}
\end{aligned}
$$


The stress tensor in the global Cartesian system may then be obtained by the simple transformation:

$$
\sigma_{i j}=\mu_{k i} \mu_{i j} \bar{\sigma}_{k t}
$$

\section{NUMERICAL EXAMPLES}

The following three-dimensional frictionless contact problems are solved using the boundary element method. Given the geometry, the elastic properties of the two bodies and external loads, the BEM is used to determine the extent of the contact region, the pressure distribution in the contact region and the stresses and displacements in the bodies.

The BEM program used for the analysis (1) is able to analyse three dimensional infinite friction and frictionless(conforming, receding and advancing) contact problems. It has been interfaced with the IDEAS package for pre-processing of the meshes and post-processing of the results. The results are compared with those obtained using the finite element method in conjunction with the IDEAS and ABAQUS packages. For the finite element analysis, linear brick elements are used to model the two bodies, and the contact conditions are modelled with gap elements which join the node-pairs.

\subsection{Elastic punch on an elastic foundation}

This case is a conforming contact and consists of an elastic punch on an elastic foundation. This is a common engineering problem, which is found in many mechanical assemblies, notably indentors. A parametric study is undertaken such that the ratio of the Young's moduli of the two bodies varies. Although according to the classical theory of elasticity the normal pressure distribution on an elastic foundation due to a punch is dependent upon the geometric profile of the punch, here only the case of a flat ended punch is considered. Fig. 6a shows an elastic punch(body B) on an elastic foundation(body A). Since the two bodies have two planes of symmetry, only one quarter of the model is analysed. 
The elastic foundation(body A) with dimensions $W_{a}=H_{a}=T_{a}=20 m m$ is lying on a rigid smooth floor, and the punch(body $B$ ) $W_{b}=W_{a} / 2=H_{b}=T_{b}=10 \mathrm{~mm}$ is located on the upper face of the foundation at its centre. Analysis is performed when the upper face of the punch is loaded by a uniform pressure with no external force nor initial relative displacement on the contact surface. The load acting uniformly over body $\mathrm{B}$ is $\mathrm{Po}=13 \mathrm{MPa}$. The boundary element discretization, deformed shape and some node labels are shown in Figs.6b and 6c, respectively. The characteristics of the mesh are recapitulated in Table 1.

Material constants of the isotropic bodies are assumed to be such that the ratio of Young's moduli, $k=E_{B} / E_{A}$, is given as 3,1 or $1 / 3$, and also 4 and 0 , implying either a rigid punch or a rigid foundation. Poisson's ratio is assumed to be $u=0.3$ for the elastic materials.

Table 2 recapitulates the normal displacements $\left(U_{y}\right)$ for some nodes in the contact zone as well as the transverse displacements $\left(U_{x}\right.$ and $\left.U_{z}\right)$. The results obtained with the boundary element method are compared to the FE results given by the ABAQUS and IDEAS packages. It can be seen that the FEM and BEM results are in good agreement. The distribution of contact traction which are in the y-direction are shown in Fig.7. The values are taken at the nodes along the diagonal line joining nodes 21 to node 13 , when $E_{B} / E_{A}$ varies between 0 and 4.

For each case the contact pressure changes moderately slowly near the central part of the contact surface, where it drops below the applied pressure, Po. By comparison it rapidly increases to higher values towards the end of the contact. If the ratio $E_{B} / E_{A}$ is less than one, then the traction distribution tends to approach the uniform applied pressure Po. On the other hand, if $E_{B} / E_{A}$ is higher than one, then the traction in the central part is smaller and tends to a higher value at the comer. Due to singularities encountered at the sharp edge of the punch contact surface, special singularity elements should be employed to give a better approximation of traction at the corner. The effect of traction singularity is always observed at the corner element, so a finer mesh can be used as the comer is approached in search of a smooth traction distribution. This effect was also noticed by Karami (10) when studying 2D contact problems by the BEM. 
To complete the study of this model a particular test is undertaken to compare these results with the results obtained by Paris (11) on a similar contact problem using the BEM. The dimensions of the punch and foundation are taken such that: $W_{b}=T_{b}=1 / 2 H_{b}=50 \mathrm{~mm}$ and $\mathrm{W}_{\mathrm{a}}=\mathrm{T}_{\mathrm{a}}=1 / 2 \mathrm{H}_{\mathrm{a}}=95 \mathrm{~mm}$. The mesh is identical to the one displayed in Fig. 6. The same properties are taken for both bodies : $E=4000 \mathrm{Mpa}$ and Poisson's ratio $\mathrm{v}=0.3$. A uniform load is acting over the punch $\mathrm{P}_{0}=1.2 \mathrm{MPa}$. In Figs. 8-9 the contact pressures obtained by Paris(11) are compared to the ones obtained by the programme developed for this study. It can be seen that the results are in good agreement. In the study performed by Paris(11), the BEM programme employs planar triangles and assuming that stresses and displacements are constant on the triangle, their values being associated to the barycentre. Due to the fact that with the linear triangular elements employed in the present paper there are not nodes along these lines, for comparison purposes the interpolated values are presented in Figs 8-9.

\subsection{Strip on an elastic foundation}

Fig. 10a shows a foundation(body A) which is lying on a rigid smooth floor, and a strip(body B) which is located on its upper face. The dimensions of the strip and the foundation are as follows: $\mathrm{L}_{a}=10 \mathrm{~mm}, \mathrm{~T}_{\mathrm{a}}=\mathrm{H}_{\mathrm{a}}=2 \mathrm{~mm}, \mathrm{~L}_{b}=8 \mathrm{~mm}, \mathrm{~T}_{b}=\mathrm{H}_{b}=1 / 2 \mathrm{H}_{\mathrm{a}}=1 \mathrm{~mm}$. The boundary element mesh and some node labels are shown in Fig. 10b. The same properties have been taken for both bodies: $E=1000 \mathrm{Mpa}$ and Poisson's ratio is assumed to be $v=0.2$ for the materials. Analysis is performed when a uniform distributed $\operatorname{load}\left(\mathrm{P}_{0}=1 \mathrm{MPa}\right)$ is applied on the upper left extremity of body $\mathrm{B}$ with no external force nor initial relative displacement on the contact surface.

A coarse mesh is used in a first instance to get a rough idea of the extent of the final contact zone once the load has been applied. The refined mesh is thereafter created in order to determine the displacements and stresses with more accuracy. The refined mesh is shown in Fig. 10b. The characteristics of the mesh is shown in Table 3. 
Table 4 recapitulates the normal displacements (Uy) for some of the nodes in the contact area. The BEM results can be compared with the FE results given by the ABAQUS and IDEAS packages. In Figs. 11(a,b\&c) the normal displacements(y-direction) are plotted. The values are taken at the nodes along the length of the strips (from $x=0$ to $8 \mathrm{~mm}$ ) and at different hights $(y=2 \mathrm{~mm}$ for nodes in the contact zone of bodies $A$ and $B$ and at $y=3 \mathrm{~mm}$ for nodes in the upper face of the strip). It appears clearly from these figures that using the BEM the refined mesh gives a better accuracy than the coarse mesh. This is more obvious for the nodes which are in the final contact zone. However, there are discrepancies between the FEM and $B E M$ results and that is due to the very fine mesh used for the FE model.

The normal stress $\left(\sigma_{y y}\right)$ distribution is presented In Fig.12. The values are taken from the nodes located in the initial contact zone which is along the complete length of strip A. It can be seen that the stress results are less accurate than the displacement results. Again the BEM results are compared with the $\mathrm{FE}$ results which are relatively in good agreement.

\section{CONCLUSIONS}

Following a brief review of the mathematical basis of the direct formulation of the BEM in linear elastostatics, a numerical formulation of this technique applied to the analysis of frictionless elastic three dimensional contact problems was presented. This method has been shown to be a very powerful tool for contact problems in two dimensions. However, due to the limitations of such models, the method was consequently extended to three dimensional problems. The numerical implementation of the BEM technique involves the use of linear triangular elements for discretising the surfaces of the bodies in contact. The treatment of the various boundary conditions was also described in details, with a particular emphasis towards the use of the appropriate contact conditions which have to be used to couple the integral equations for the bodies involved. Having solved the coupled system of equations, the required variables for each body could be obtained from the solution vector. A computer program based on this numerical formulation, was developed for use in this study. 
An iterative procedure was developed in order to define the extent of the contact zone by finding a boundary solution compatible with the contact conditions (no interpenetrations between the domains outside the contact zone and no tractions on the final contact zone). The frictionless contact and infinite friction contact conditions were studied. For the first case sliding conditions are always assumed, in contrary to the latter where the node-pairs are stuck together. Another distinction is finally made concerning the frictionless contact problem which in function of the evolution of the initial contact zone, after the application of the load, can be termed as conforming advancing or receding. For the conforming condition no iteration is required in contrary to the two other cases. The iterative procedure was preferred to the incremental one which was shown (in the literature) to be more costly than a trial and error iterative procedure. However this consideration would be different with the presence of friction, due to its irreversible character and to the possibility of appearances of local unloadings.

Two examples have been presented to demonstrate some of the features of the solution procedure. The results have been compared with those obtained using the finite element method. Two FE packages have been used, I-DEAS and ABAQUS. For the case of the frictionless conforming contact, the results obtained for the diverse examples treated were in good agreement with those given by the FEM. However, the percentage of error when comparing the stresses were always noticed to be higher than when the displacements were compared. The test case involving a punch on a foundation shows how different material properties can influence the stresses generated. It appeared clearly that for comer elements some singularities in the stresses occur. The inability of a coarse mesh to reproduce the correct singularity was quite apparent. This singularity is highly dependent on the ratio of the Young's moduli of the two bodies in contact. A finer mesh could be used at the comer in search of a smooth traction distribution but some form of singular elements would be preferable. The final results in what is determined as the final contact zone after that the iterative procedure has been completed are generally found to be in good agreement with $\mathrm{FE}$ results. 
Consequently and with the limited results obtained it appears that the BEM is well suited to the analysis of contact problems. Since in most contact problems only the boundary values are needed and no internal discretisation is required, therefore, attention can be concentrated on the small area of contact. Finally the method allows direct solutions for the displacements and tractions in the contact area. Consequently the mesh design and data preparation processes can be simplified, and the computer storage requirements reduced in comparison to the finite element method. These advantages become even more apparent for contact problems, where more than one body is analysed.

\section{ACKNOWLEDGEMENTS}

The authors would like to thank the University of Manchester for the allocation of Simon Scholarship to support this work. The authors also would like to thank Professor R.T.Fenner from the Imperial College of Science, Technology and Medicine to let them to use the threedimensional boundary element $\operatorname{code}(25)$ for elastostatic stress analysis as the "building block". In this study the building block programme was further developed to employ linear triangular elements for mesh discretization and cover all the aforementioned procedures to solve three-dimensional contact problems (1). 
Segond, D. \& Tafreshi, A. Oct 1998 In : Engineering Analysis with Boundary Elements. 22, 3, p. 199-214 16 p.

\section{REFERENCES}

1) Segond, D., "Stress Analysis of Three-Dimensional Contact Problems Without Friction Using the Boundary Element Method", MPhil Thesis, Manchester University, 1997

2) ABAQUS User's Manual, Version 5.6, Hibbit, Karlson \& Sorenson Inc., 1997

3) IDEAS(Integrated Design Engineering Software), Structural Dynamics Research Corporation), USA, 1996

4) Gladwell, G.M.L., "Contact Problems in the Classical Theory of Elasticity", Sijthoff \& Noordhoff, 1980

5) Johnson, K.L., "Contact Mechanics", Cambridge University Press, 1985

6) Munisamy, R.L., Hills, D.A., Nowell, D., "A Numerical Analysis of an Elastically Dissimilar Three-Dimensional Sliding Contact”, Proc. Instn. Mech. Engrs, Vol 206 (Part C:J.Mech. Eng. Science), pp203-211, 1992

7) Okamoto, N. and Nakazawa, M. "Finite Element Incremental Contact Analysis with Various Frictional Conditions", Int. J. Numer. Meth. Engng, V14, pp337-357, 1979

8) Hung, N.A. and de Saxe, "Frictionless Contact of Elastic Bodies by Finite Element Method and Mathematical Programming Technique", Computers \& Structures, 11, pp55-67, 1980

9) Tafreshi, A. and Dover, W.D., "Stress Analysis of Drillstring Threaded Joints Using the Finite Element Method", Int. J. of Fatigue, V15, N5, pp429-438, 1993

10) Karami, G., "A Boundary Integral Equation Method for Two-Dimensional Elastic Contact Problems", PhD Thesis, Imperial College of Science and Technology, University of London, 1983

11) Paris, F., Foces, A. and Garrido, J.A., "Application of Boundary Element Method to Solve Three-Dimensional Elastic Contact Problems Without Friction", Computers and Structures, 43, pp 19-30, 1992

12) Foces, A., Garrido, J.A. and Paris, F., "Three-Dimensional Contact Using BEM". In: Computational Method in Contact Mechanics (Edited by M.H.Aliabadi and C.A. Brebbia), pp 191-232, 1993

13) Paris, F., Blazquez. A. and Canas, J., "Contact Problems With Nonconforming 
Discretization using the Boundary Element Method", Computers and Structures, No. 5, pp829-839, 1995

14) Anderson, T., "The Second Generation Boundary Element Contact Problems". In Proc. of the third International Seminar on Recent Advances in Boundary Element Methods", Irvine, California, C.A. Brebbia(Ed.), Southampton, 1982

15) Anderson, T. and Allan-Persson, "The Boundary Element Method Applied to Twodimensional Contact Problems with friction", In Proc. of the Third International Seminar on Recent Advances in Boundary Element Methods", Irvine, California, C.A.Brebbia(Ed.), Springer-Verlag, Berlin, 1981

16) Abdhul-Mishein, M.J., Becker, A.A., and Parker, A.P., "A Boundary Integral Equation Method for Axi-symmetric Elastic Contact Problems" Computers and Structures, 23, 787-793, 1986

17) Becker, A.A., and Plant, R.C.A., "Contact Mechanics using the Boundary Element Method", Proc. Int. Conf. on Tribology, Friction, Lubrication and Wear, The Institution of Mechanical Engineering, London, 1987

18) Paris, F. and Garrido, J.A., "On the Use of Discontinuous Elements in Twodimensional Contact Problems", in Boundary Element VII, C.A. Brebbia(Ed.), Springer, 1985

19) Paris, F, and Garrido, J.A., "Friction Multicontact Problems with BEM", in Boundary Element X, C.A.Brebbia(Ed.), Springer, 1988

20) Dandekar, B.W. and Conant, R.J., "Numerical Analysis of Elastic Contact Problems Using the Boundary Integral Equation Method. Part I: Theory", Int. J. Num. Meth.: Engng. 33, 1513-1522, 1992

21) Dandekar, B.W., and Conant, R.J., "Numerical Analysis of Elastic Contact Problems Using the Boundary Integral Equation Method. Part II: Results", Int. J. Num. Meth. Engng, 33, 1523-1535, 1992

22) Tafreshi, A. and Fenner, R.T., "Design Sensitivity Analvsis using the Boundary Element Method", J. Strain Analysis, V28, N4, pp 283-291, 1993

23) Tafreshi, A. and Fenner, R.T. "Design Optimization Using the Boundary Element Method", J. Strain Analysis, V26, N4, pp231-241, 1991

24) Keer, L.M., Dundurs, j. and Tsai, K.C., "Problems Involving a Receding Contact 
Segond, D. \& Tafreshi, A. Oct 1998 In : Engineering Analysis with Boundary Elements. 22, 3, p. 199-214 16 p.

Between a Laver and a Half Space", J. Appl. Mech., ASME, 39, pp1115-1120, 1972

25) Tan, C.L., "Three-Dimensional Boundary Integral Equation Stress Analysis of Cracked Components", $\mathrm{PhD}$ Thesis, Imperial College of Science and Technology, University of Londion, 1979

26) Lachat, J.C. and Watson, J.O., "Effective Numerical Treatment of Boundary Integral Equation: A Formulation for Three-Dimensional Elastostatics", Int. J. Num. Meth. Engng, 10, pp991-1005, 1976

27) Watson, J.O., "Advanced Implementation of the Boundary Element Mehod for Twoand Three-Dimensional Elastostatics", Development in Boundary Element Methods I, (eds. P.K.Banerjee and R.Butterfield), Applied Science Publishers, London, 1979 
Table 1 Mesh characteristics(Example 8.1)

\begin{tabular}{ccc}
\hline Body & A & B \\
\hline Number of elements & 224 & 80 \\
Number of nodes & 114 & 42 \\
No.Elements in the contact zone & 16 & 16 \\
\hline
\end{tabular}

Table 3 Mesh characteristics(Example 8.2)

\begin{tabular}{ccl}
\hline Body & A & B \\
\hline Number of elements & 158 & 84 \\
Number of nodes & 81 & 44 \\
No.Elements in the contact zone & 30 & 30 \\
\hline
\end{tabular}

Table 4 Normal displacements $\left(\mathrm{y}\right.$-dir) $\left(\mathrm{U}_{\mathrm{y}} \times 10^{-4} \mathrm{~mm}\right)$ of some nodes in the final contact zone, (Example 8.2)

\begin{tabular}{cccc}
\hline Node & BEM & ABAQUS & DEAS \\
\hline 16 & -7.87 & -7.74 & -7.79 \\
17 & -5.45 & -5.29 & -5.35 \\
19 & -16.7 & -17 & -17.91 \\
20 & -11.9 & -11.84 & -12.53 \\
\hline
\end{tabular}


Table 2 Displacements in the contact zone analysed using the BEM and FEM(in conjunction with the ABAQUS and DEAS packages),(Example 7.1)

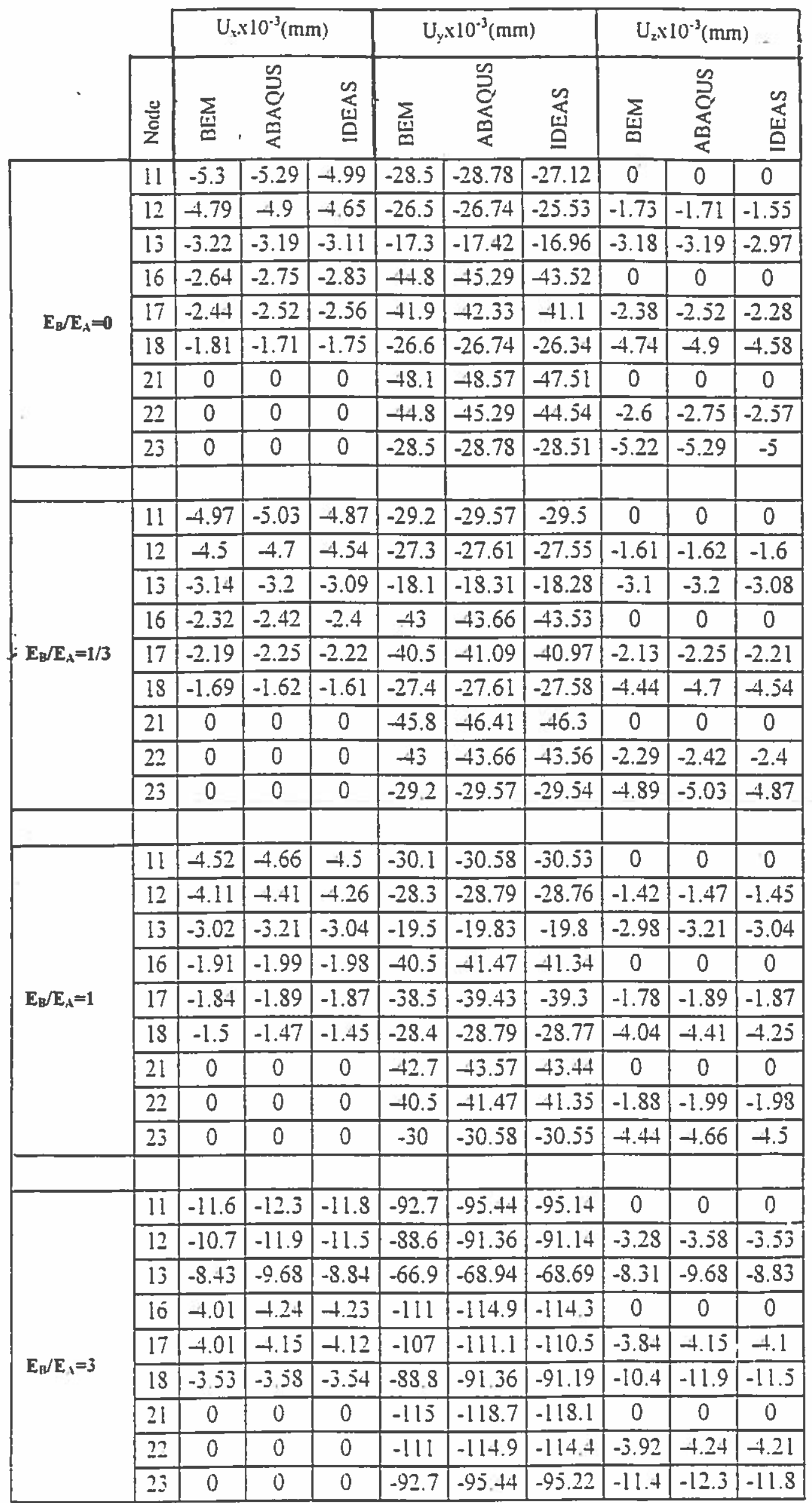




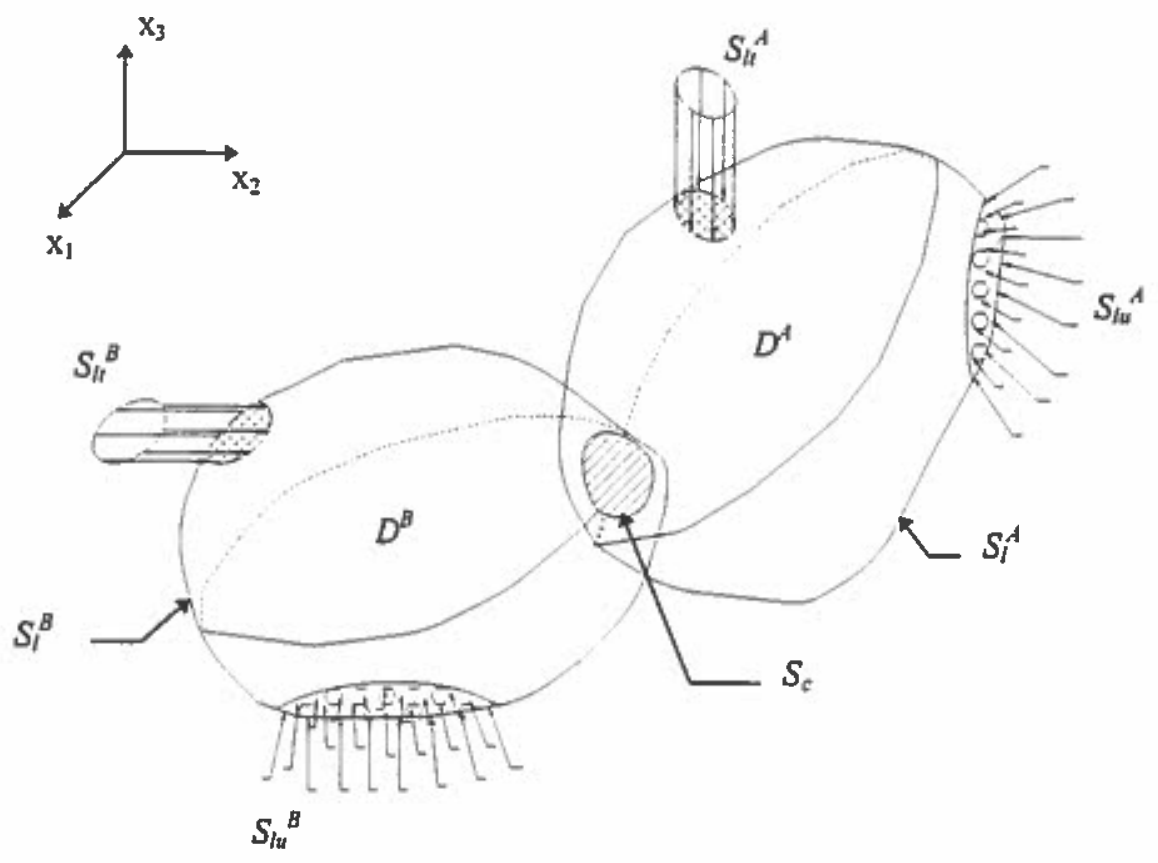

Figure 1 Contact problem definition 


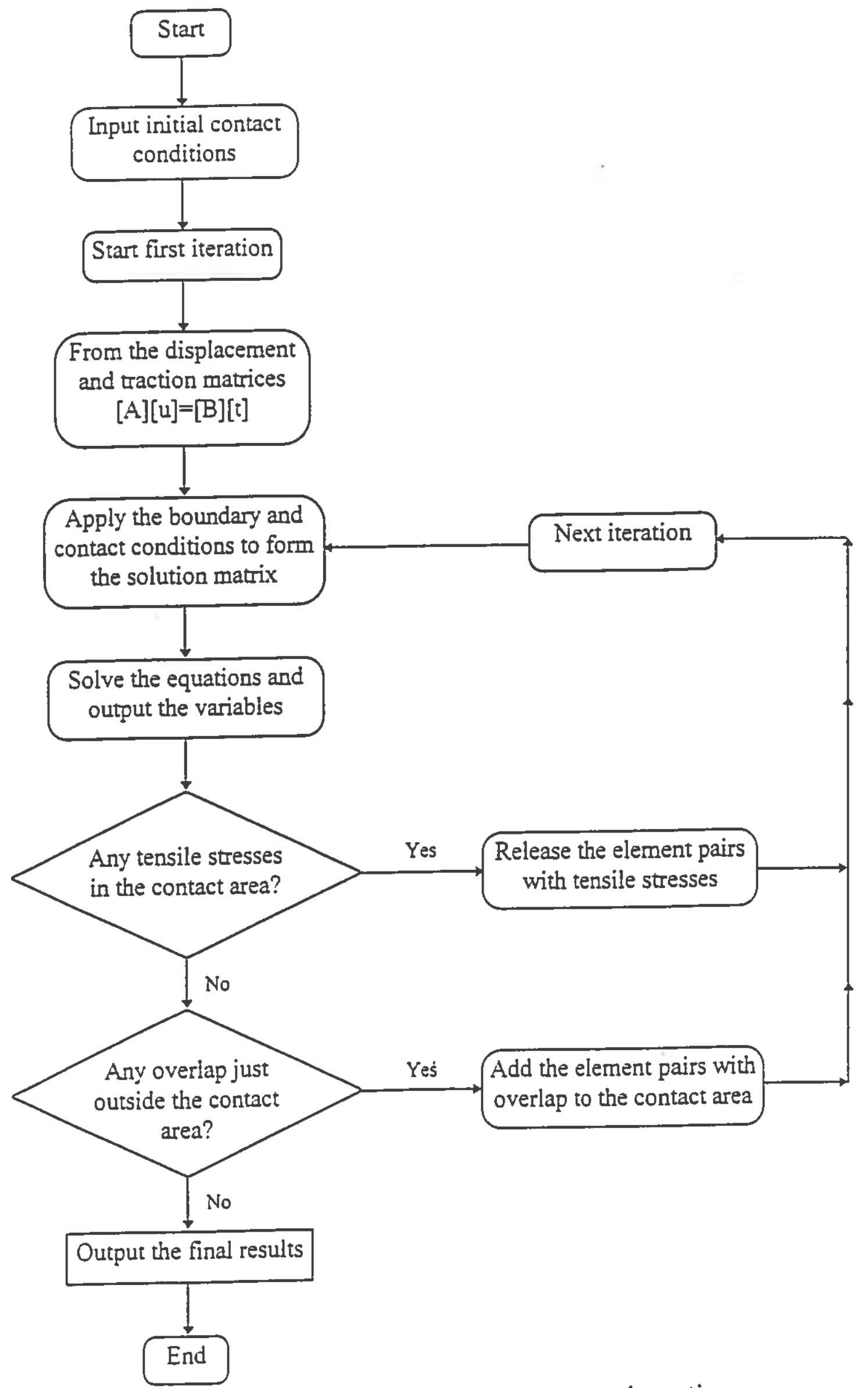

Figure 2 Flow chart for the frictionless contact iterations 


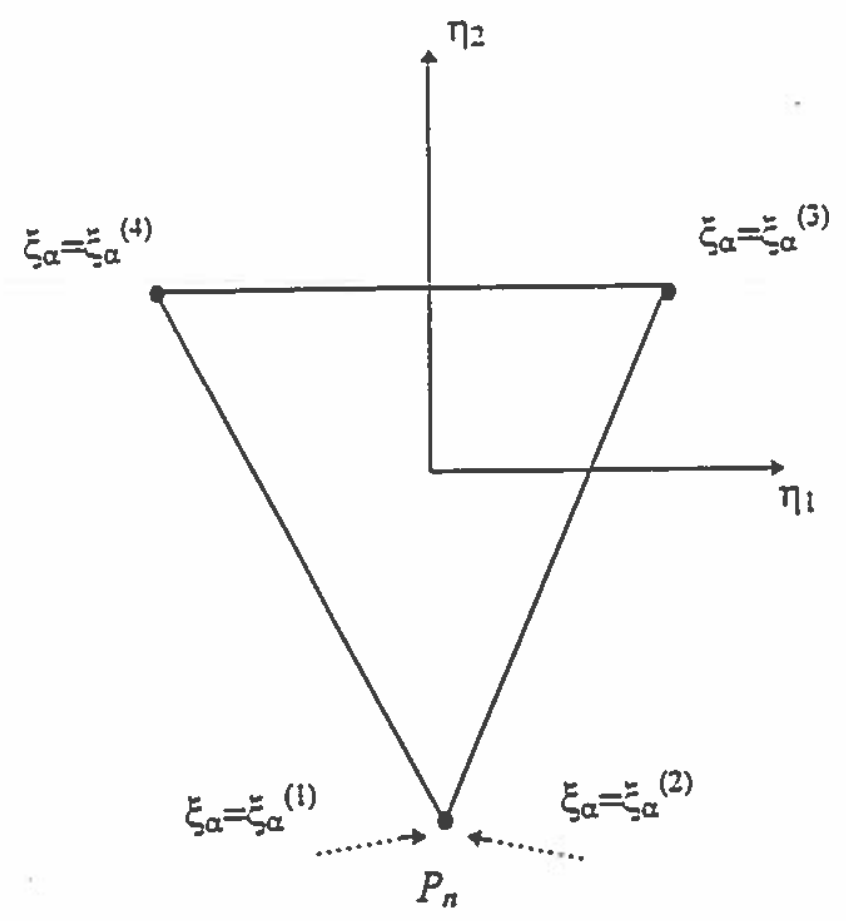

Figure 3 Local intrinsic coordinate system for singularity removal 


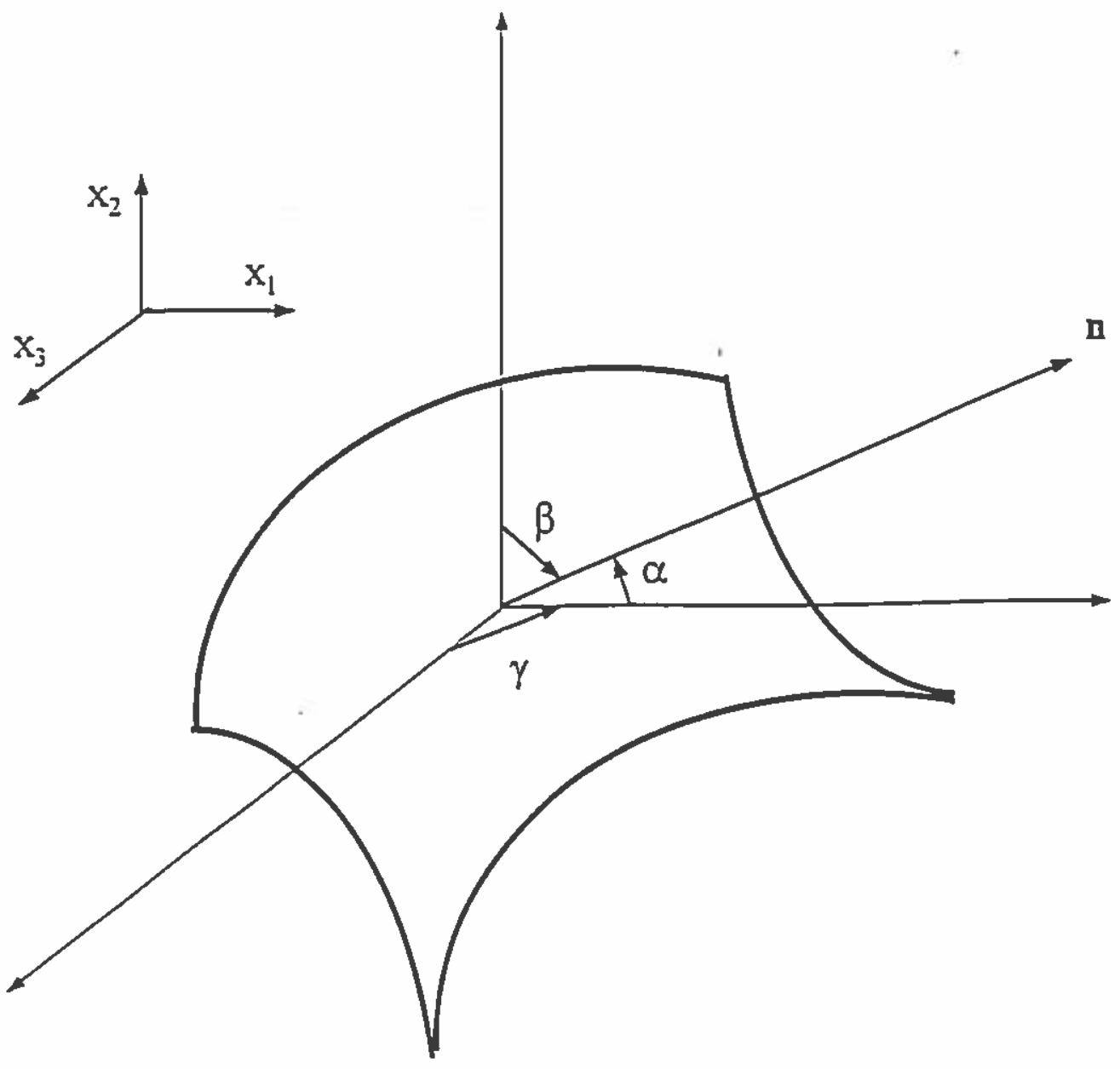

Figure 4 Global coordinate system 


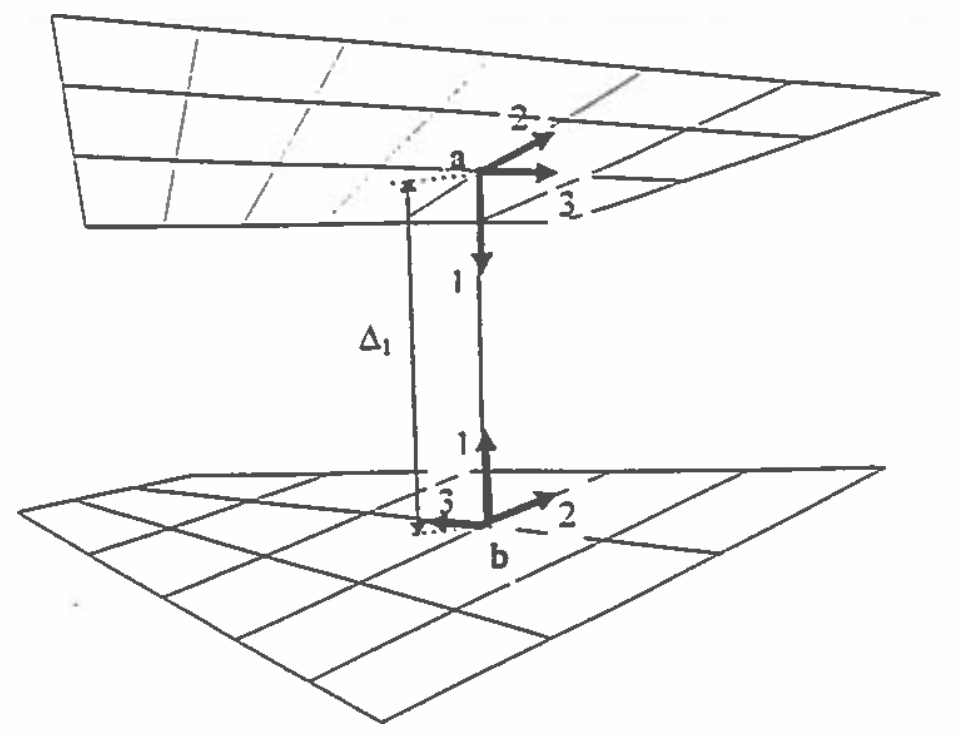

Figure 5 Definition of the local system of reference 


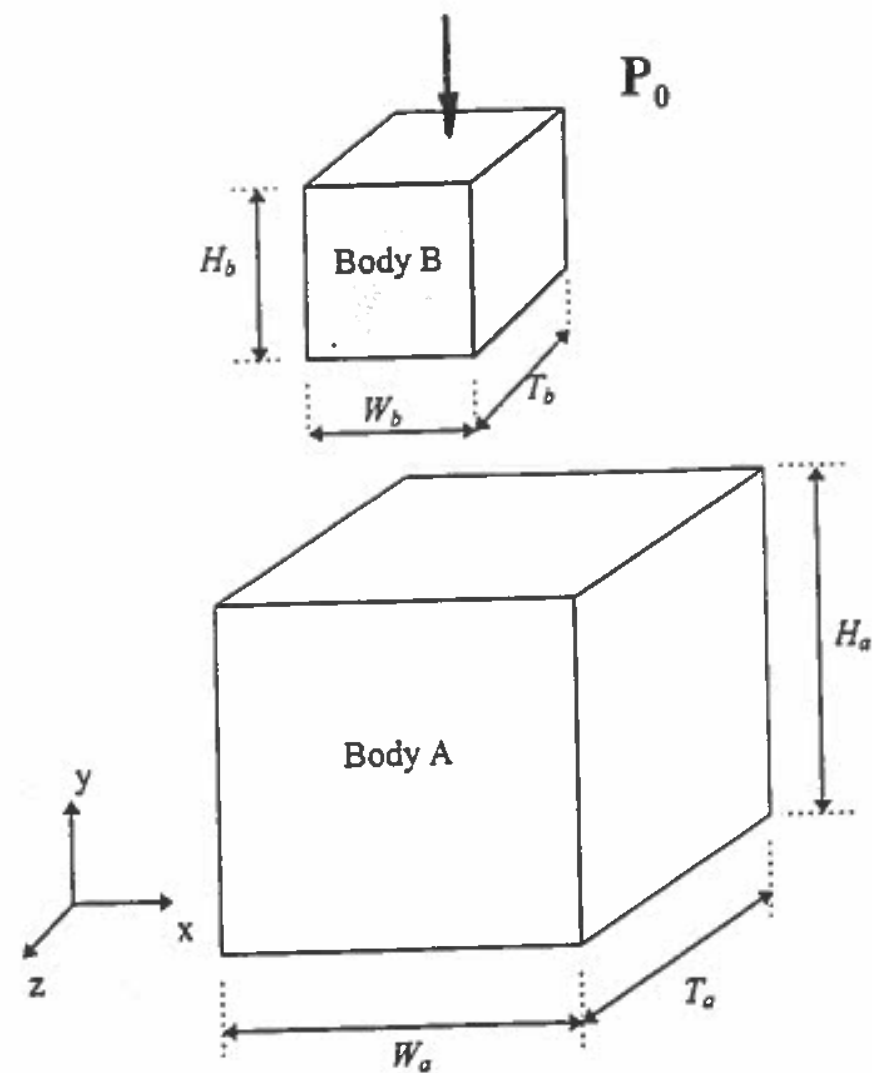

(a)

(c)

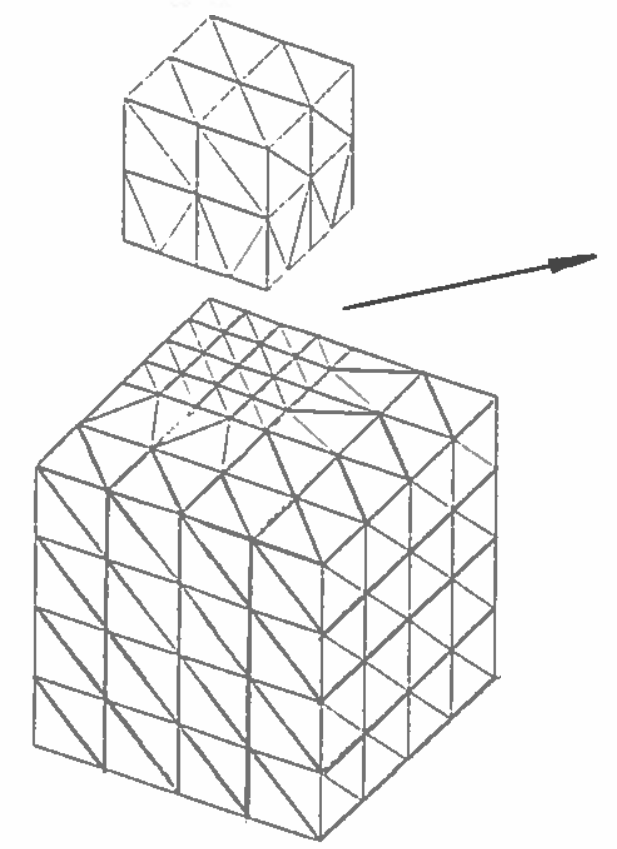

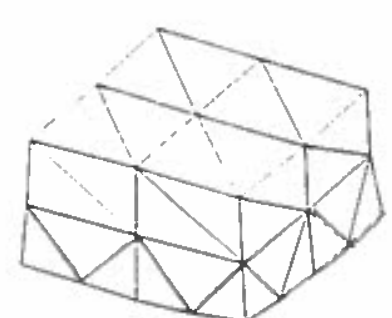

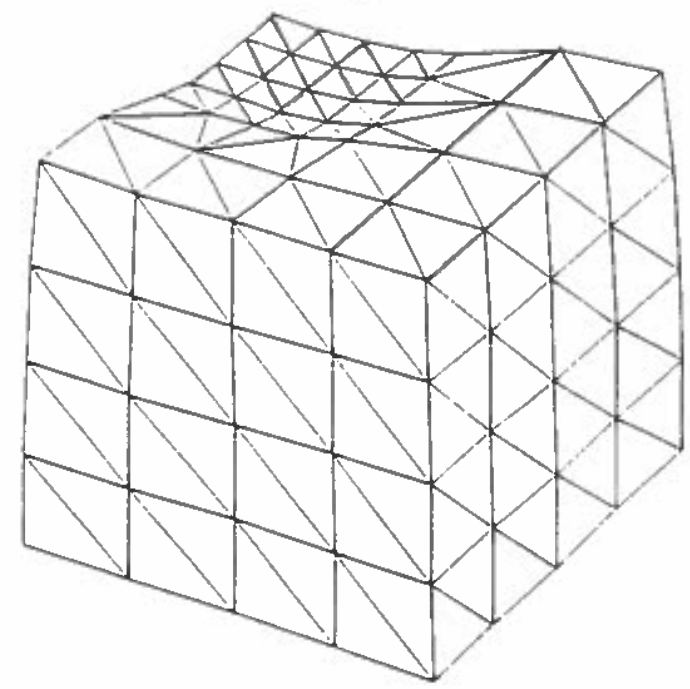

(b)

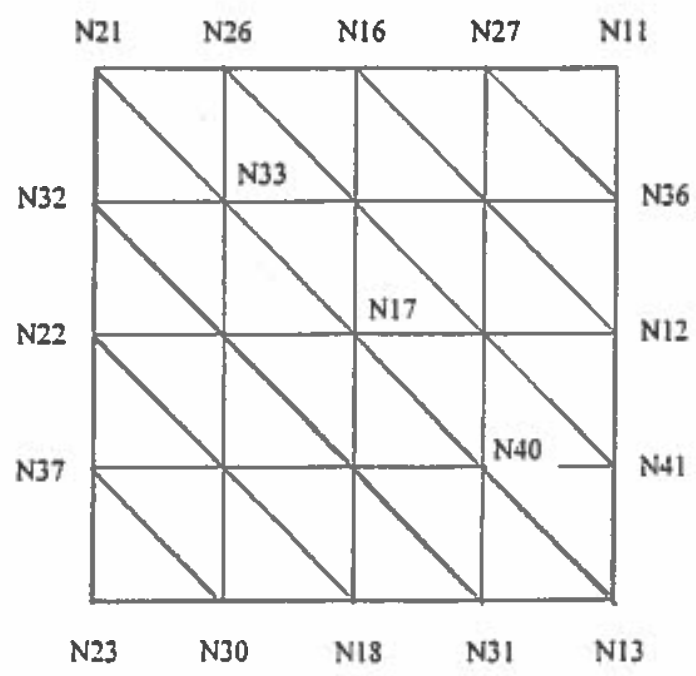

Fig. 6 (Example 7.1): a) Problem definition

b) Deformed geometry

c) Discretization of the contact zone 


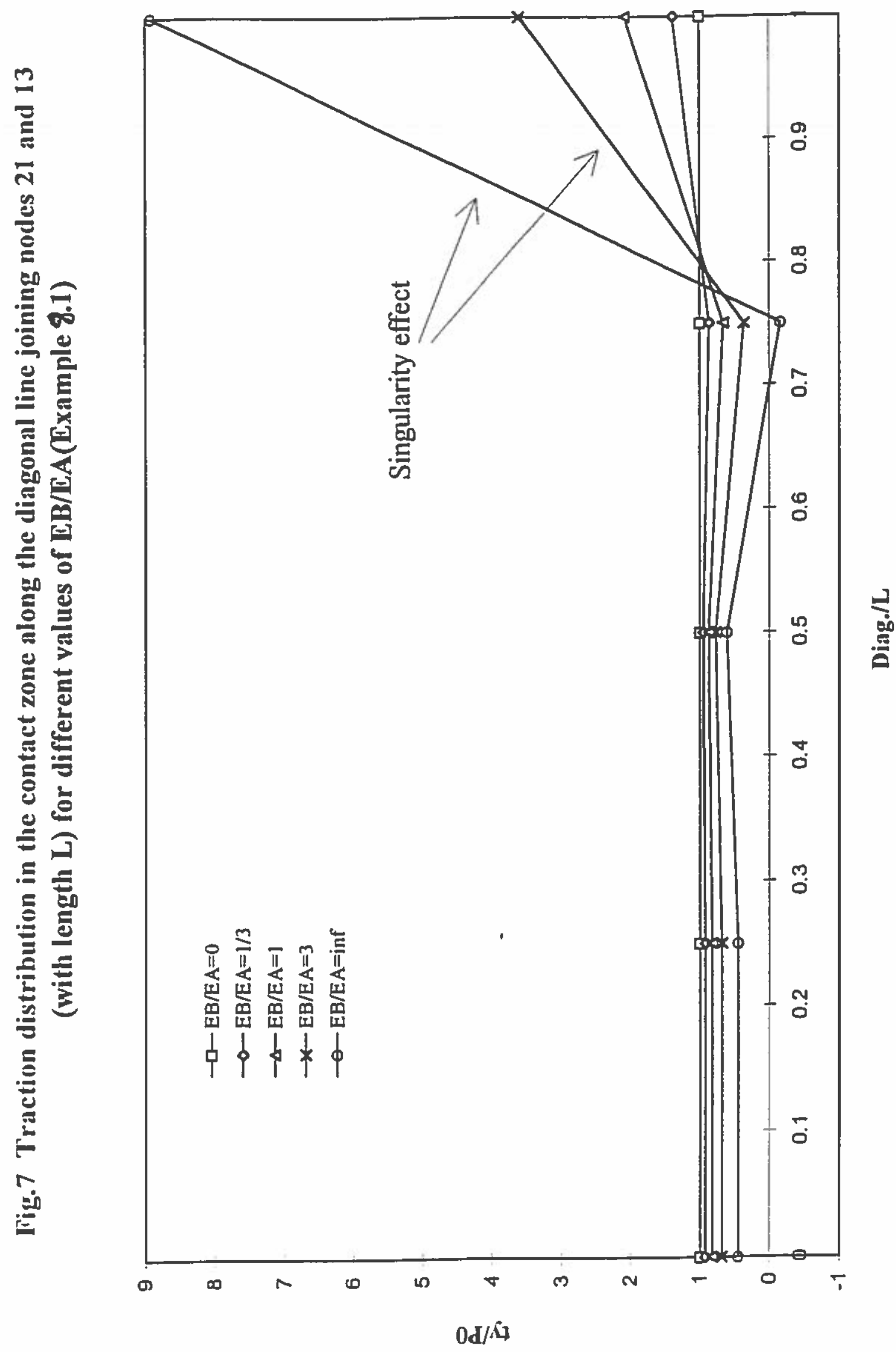


Segond, D. \& Tafreshi, A. Oct 1998 In : Engineering Analysis with Boundary Elements. 22, 3, p. 199-214 16 p.

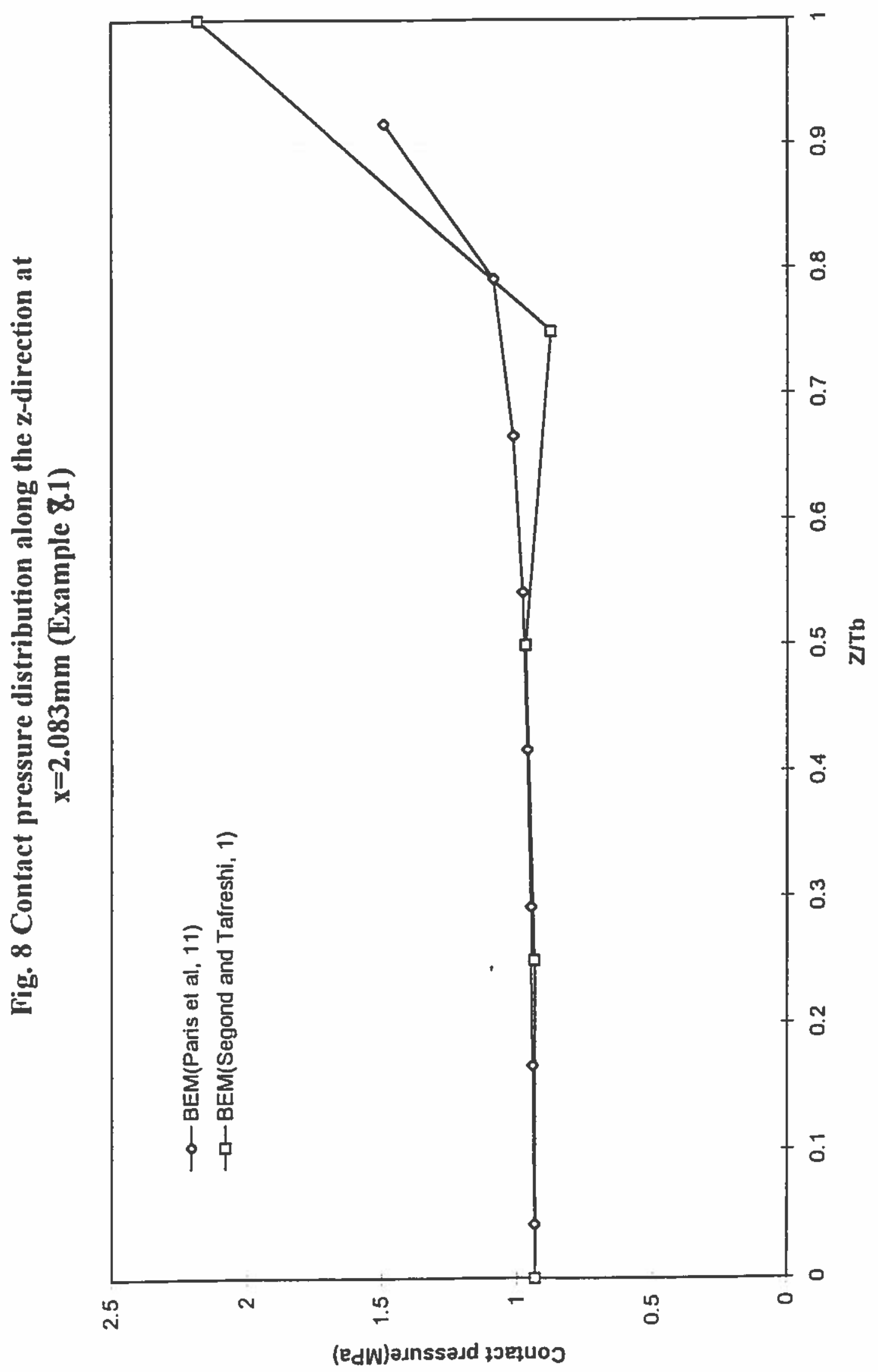




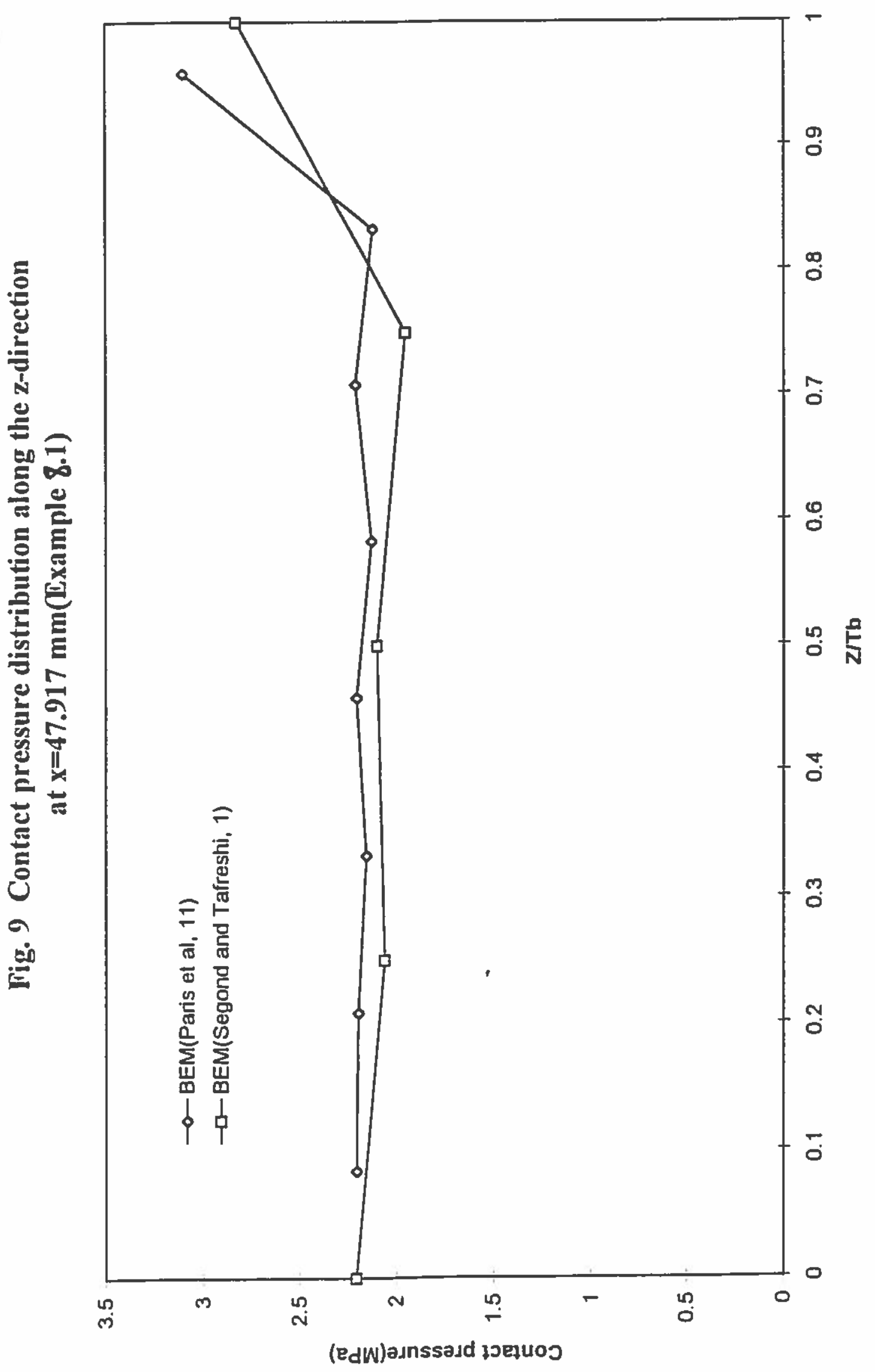


Segond, D. \& Tafreshi, A. Oct 1998 In : Engineering Analysis with Boundary Elements. 22, 3, p. 199-214 16 p.

(a)

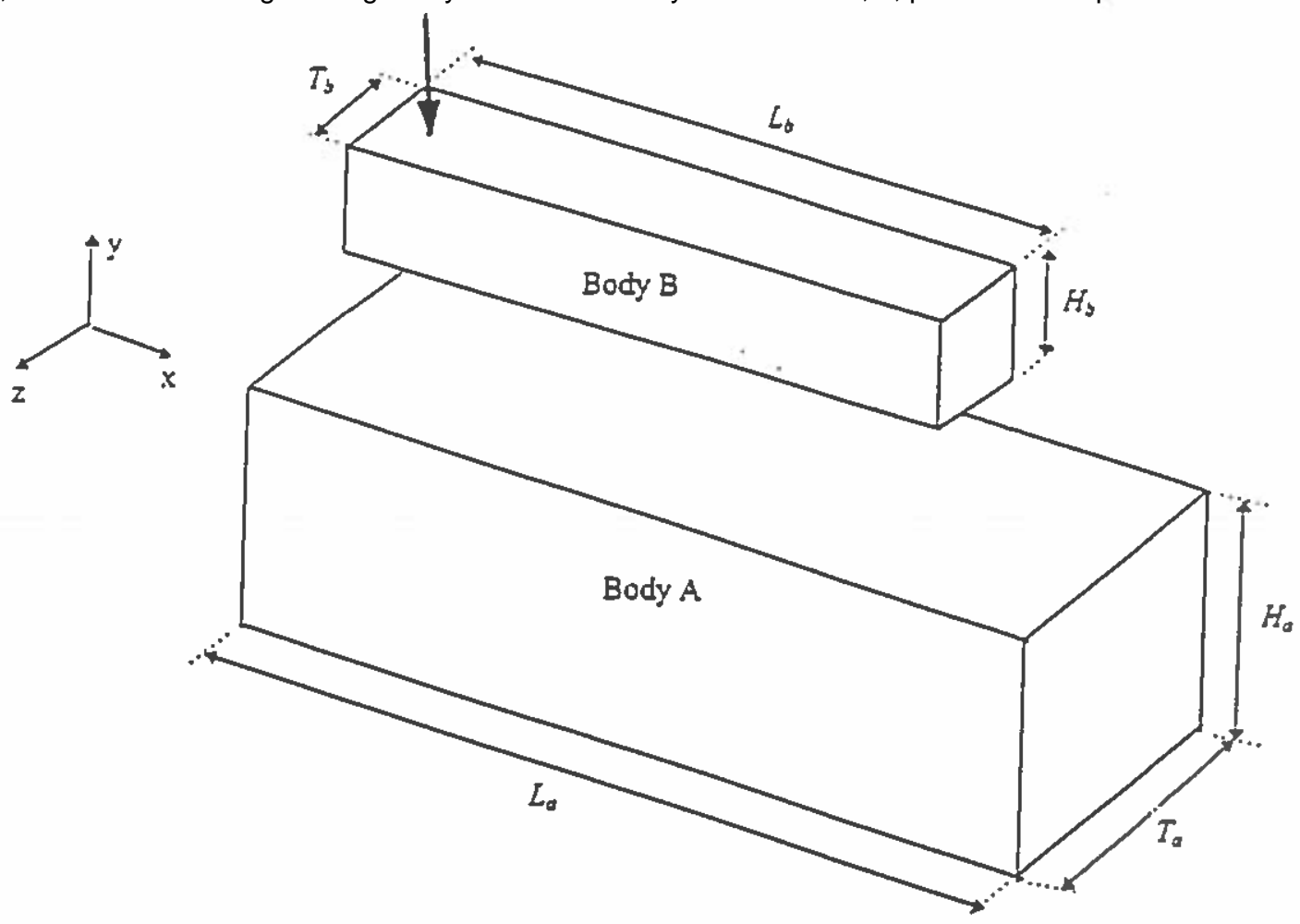

(c)

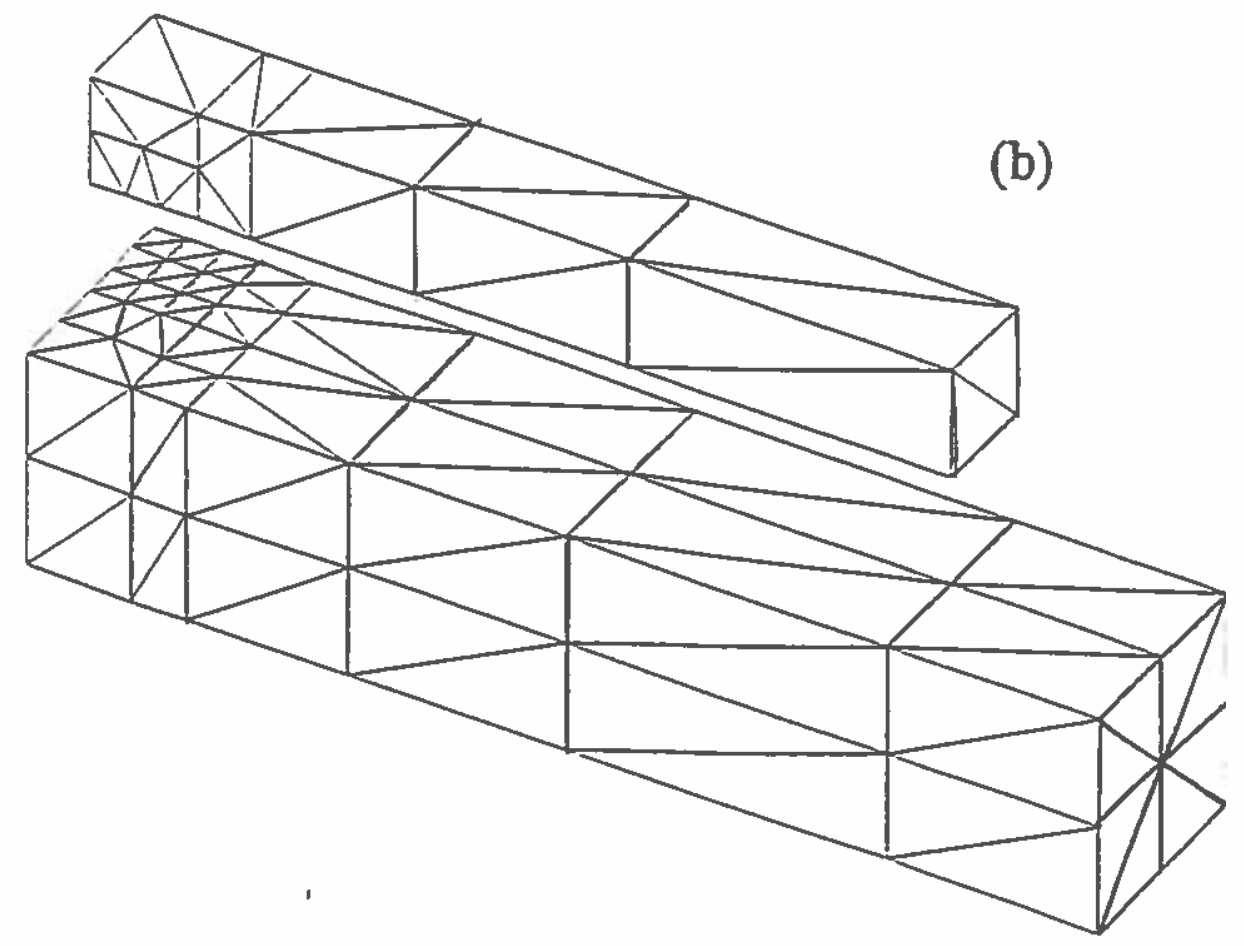

Fig. 10 (Example 7.2): a) Problem definition

b) Mesh discretization

c) Elements on the contact face of body $B$ 


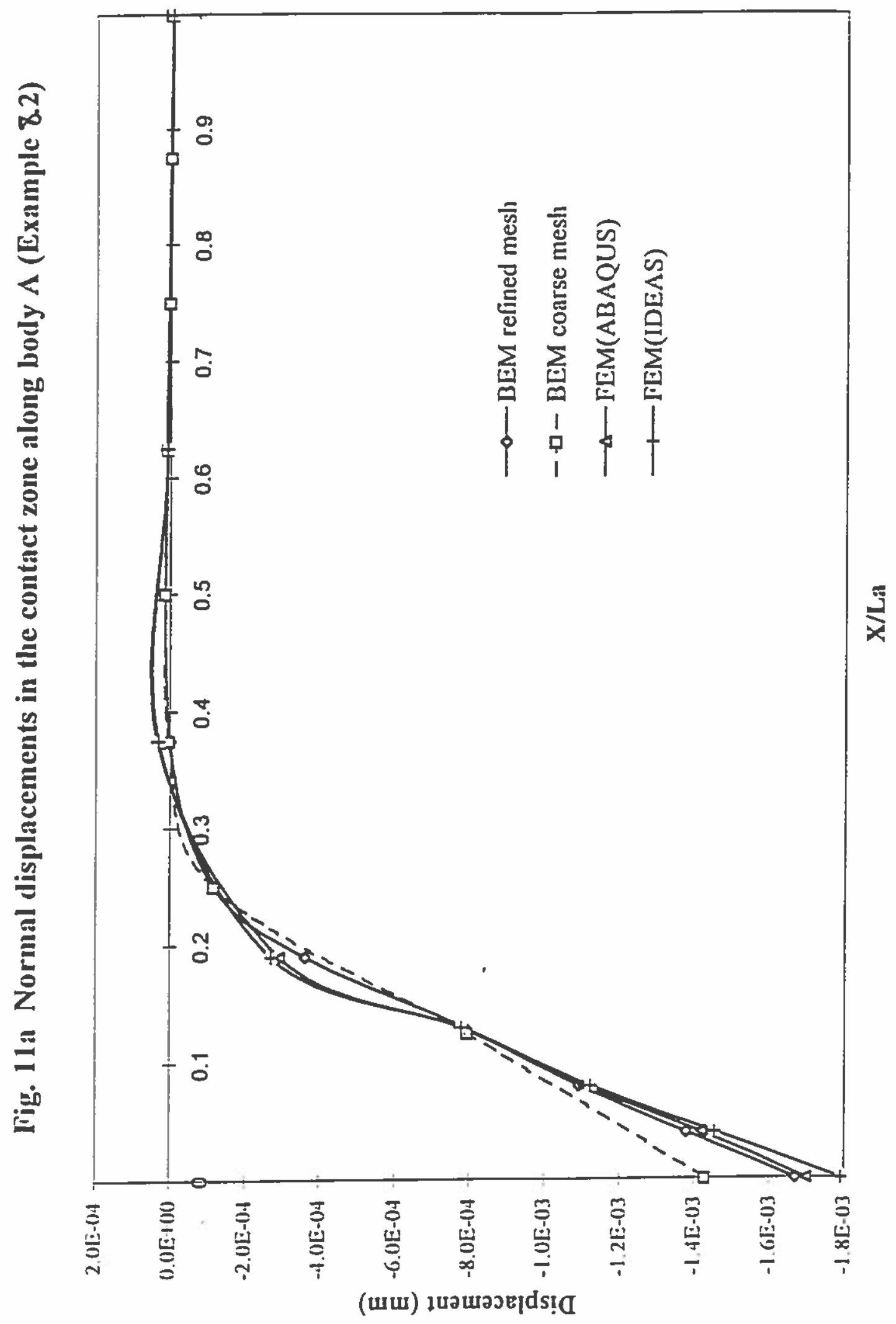




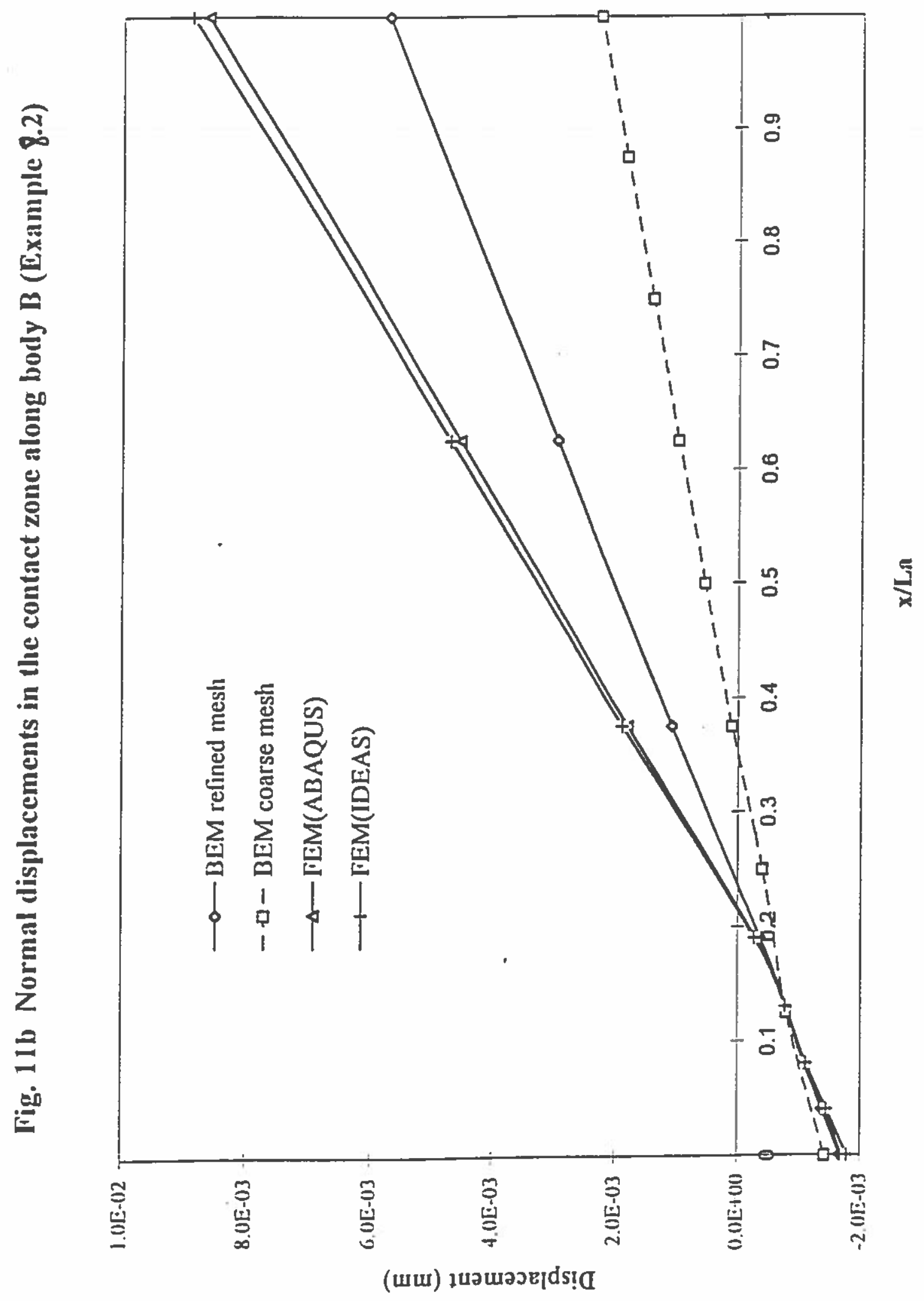




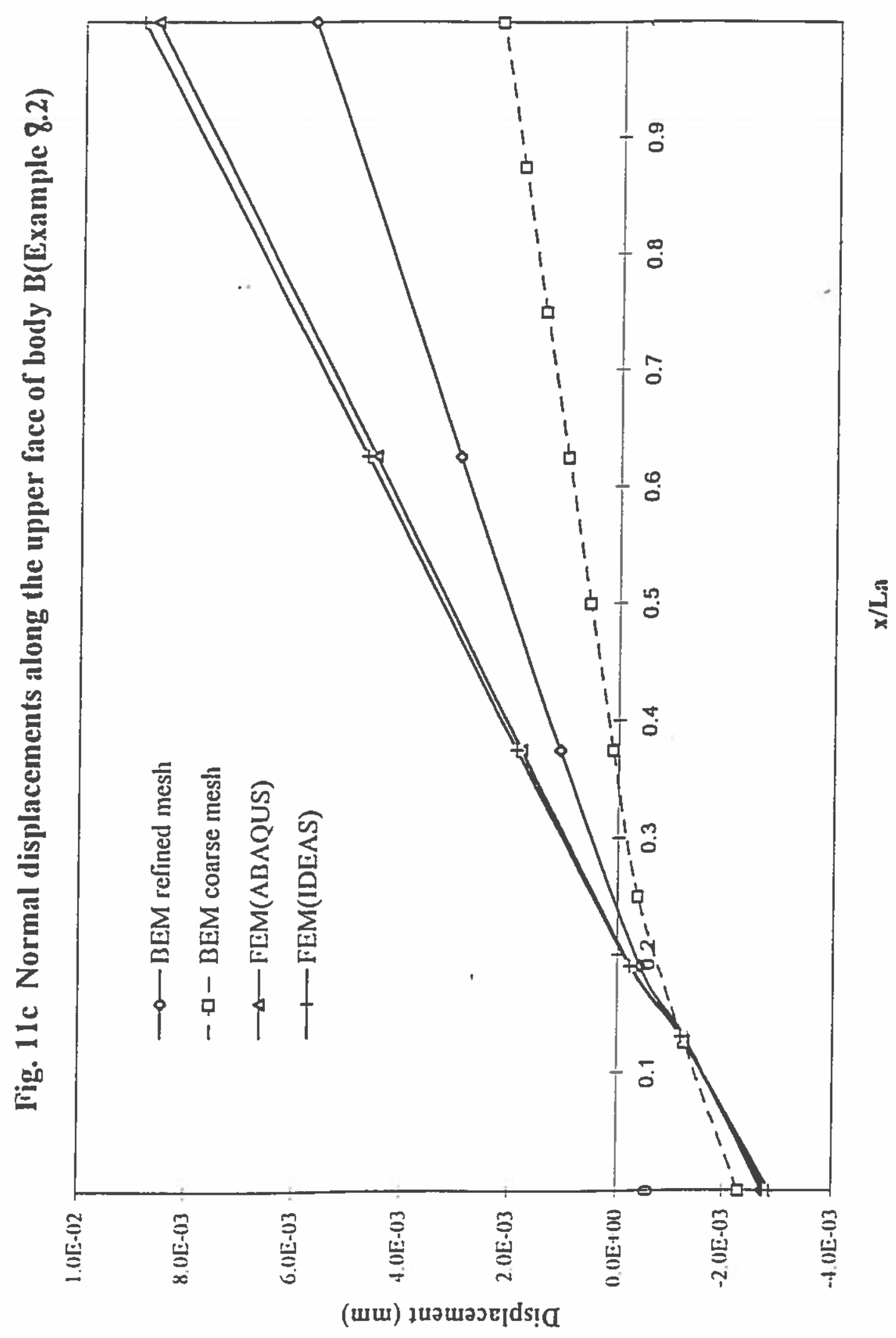




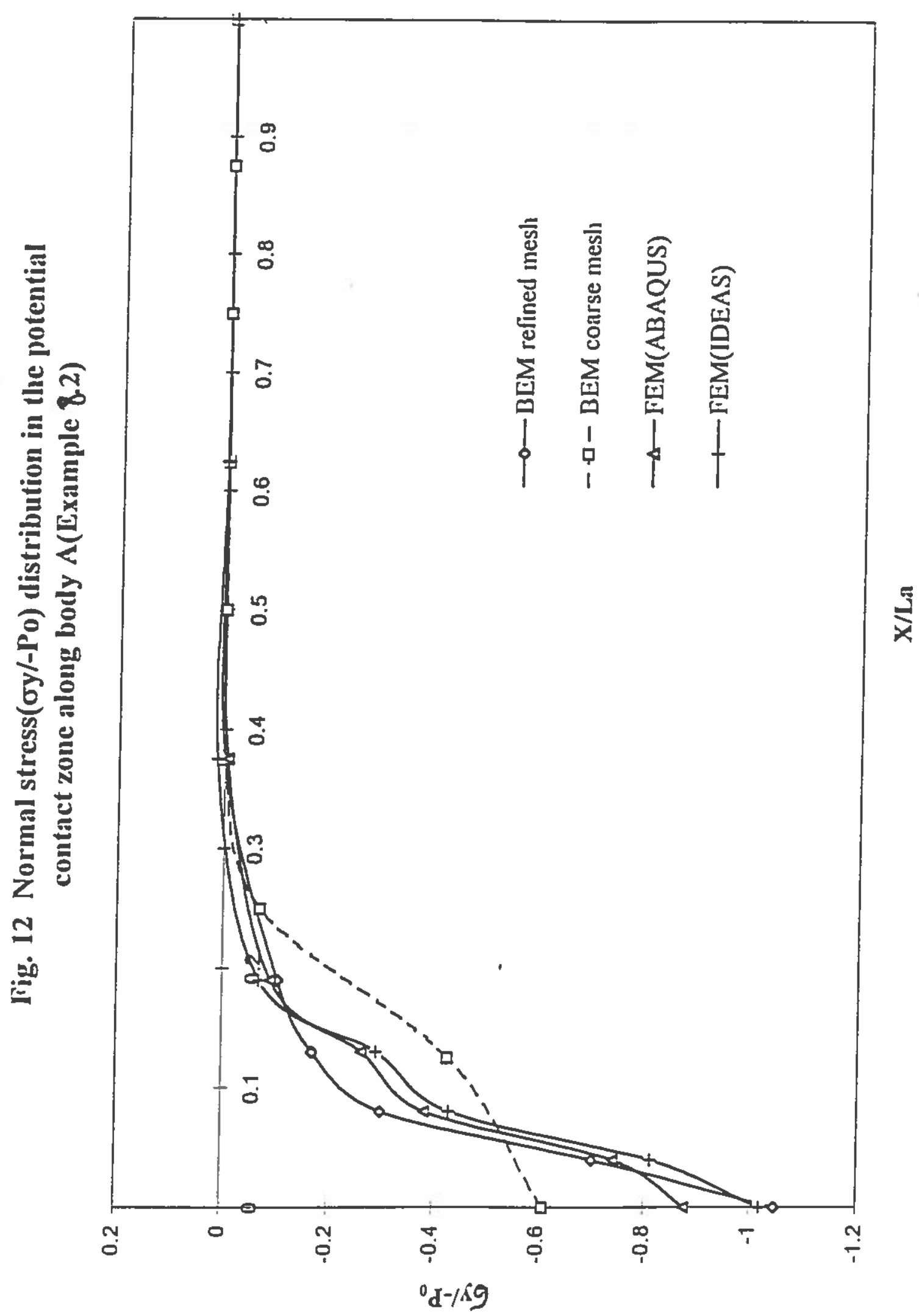

